
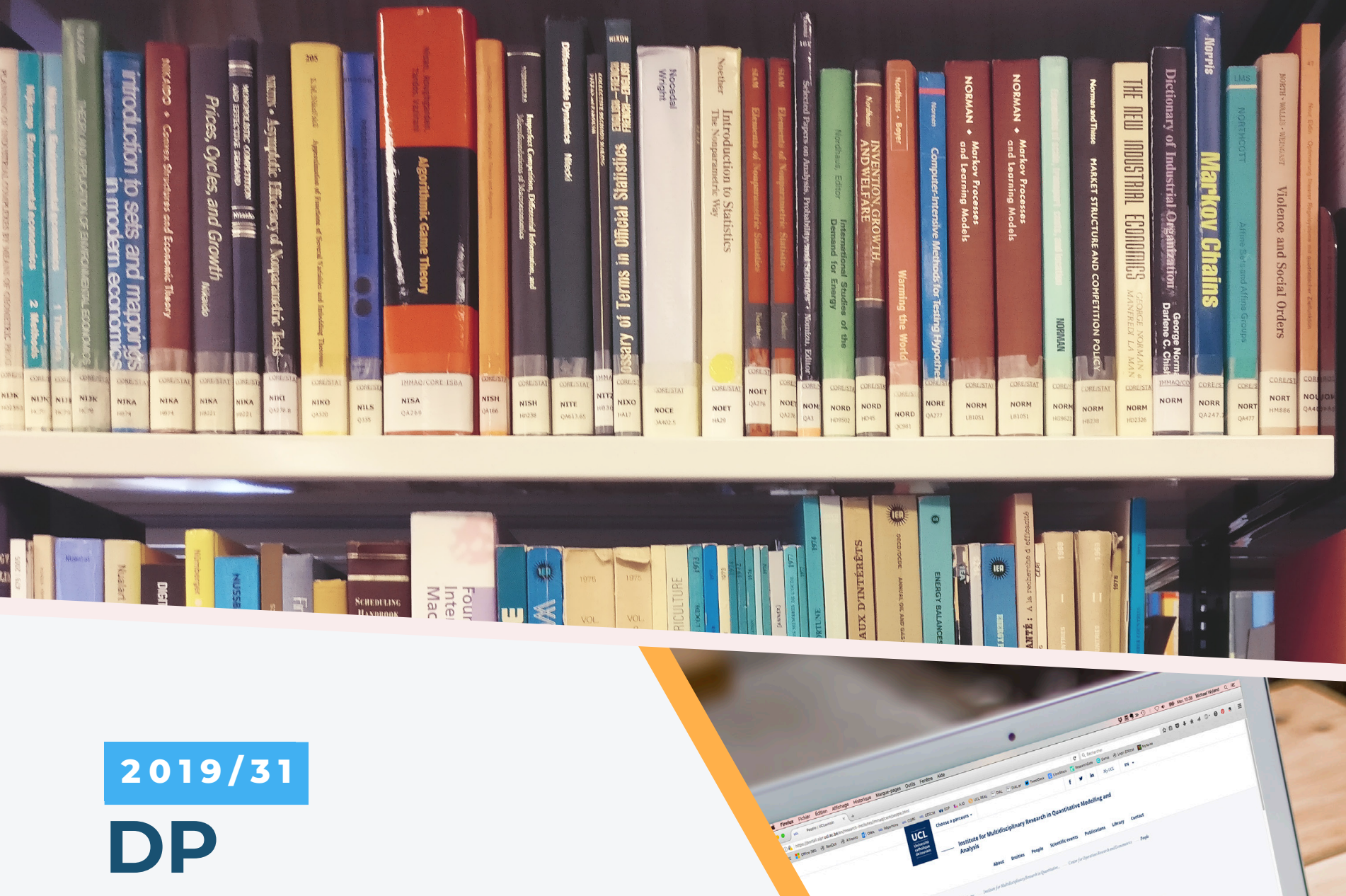

Christian M. Hafner and Linqi Wang

A dynamic conditional score model for the log correlation matrix 


\section{CORE}

Voie du Roman Pays 34, L1.03.01

B-1348 Louvain-la-Neuve

Tel (32 10) 474304

Email: immaq-library@uclouvain.be

https://uclouvain.be/en/research-institutes/

lidam/core/discussion-papers.html 


\title{
A dynamic conditional score model for the log correlation matrix
}

\author{
Christian M. Hafner*and Linqi Wang ${ }^{\dagger}$
}

December 18, 2019

\begin{abstract}
This paper proposes a new model for the dynamics of correlation matrices, where the dynamics are driven by the likelihood score with respect to the matrix logarithm of the correlation matrix. In analogy to the exponential GARCH model for volatility, this transformation ensures that the correlation matrices remain positive definite, even in high dimensions. For the conditional distribution of returns we assume a student-t copula to explain the dependence structure and univariate student-t for the marginals with potentially different degrees of freedom. The separation into volatility and correlation parts allows two-step estimation, which facilitates estimation in high dimensions. We derive estimation theory for one-step and twostep estimation. In an application to a set of six asset indices including financial and alternative assets we show that the model performs well in terms of various diagnostics and specification tests.
\end{abstract}

Keywords: score, correlation, matrix logarithm, identification

JEL classification: C14, C43, Z11

*ISBA/CORE and Louvain Institute of Data Analysis and Modelling, Université catholique de Louvain, christian.hafner@uclouvain.be

${ }^{\dagger}$ LFIN and Louvain Institute of Data Analysis and Modelling, Université catholique de Louvain, linqi.wang@uclouvain. be. 


\section{Introduction}

Correlation matrices are pervasive in many applied sciences such as finance and econometrics. Reliable models for the dynamics of correlations between different assets or underlying risk factors are essential for asset pricing, portfolio selection and risk management. Nonetheless, correlation matrices are notoriously difficult to model in high dimensions, for at least two reasons: the positivity constraint and the balance between flexibility and computational feasibility. These challenges are well documented and addressed in the framework of multivariate GARCH models, see e.g. Bauwens et al. (2006), Bauwens et al. (2012) and Francq and Zakoian (2019).

This paper proposes a new model for the joint modelling of volatilities and correlations, which both are driven by the respective conditional scores of the likelihood, and hence fits into the general modelling framework of dynamic conditional score or, in an alternative terminology, generalized autoregressive score models, as introduced by the seminal works of Creal et al. (2011), Creal et al. (2013) and Harvey (2013). Score driven correlation models have been proposed recently in the context of modelling realized variances and covariances. For example, Gorgi et al. (2018) use the Wishart distribution, and Opschoor et al. (2018) and Vassallo et al. (2018) the more flexible matrix-F distribution.

Our aim is to allow for fat tails in the conditional distributions, and in particular, to generalize the popular Beta-t-EGARCH model proposed in Harvey (2013) (Section 4.1), which uses the student-t distribution, to the multivariate case. The conditional covariance matrix can be decomposed into volatility and correlation components, which facilitates estimation since it can be split into two parts as in the DCC model of Engle (2002) and in Vassallo et al. (2018).

For the correlation part, in order to satisfy the positivity constraint in arbitrary dimensions, we specify the dynamics for the matrix logarithm of the correlation matrix. This is analogous to modelling the log volatility for Beta-tEGARCH which ensures positivity of volatilities. By construction, the matrix exponential of the specified log correlation matrix will be positive definite. The parameter characterizing the dynamics of the log 
correlation matrix will be driven by the score of the likelihood with respect to this parameter. The distribution characterizing the dependence part of the model is assumed to be a student-t copula. If all conditional marginal distributions happen to be univariate student-t with the same degrees-of-freedom, equal to the parameter of the t-copula, then we have a multivariate student-t distribution. However, we do not impose this restriction, so we can have what is commonly called a Meta-t distribution, see Demarta and McNeil (2005), i.e. a student-t copula combined with arbitrary student-t marginals.

The idea of modelling the matrix log of a correlation or covariance matrix is not new. For example, Kawakatsu (2006) proposed a multivariate version of the EGARCH model of Nelson (1991), but with ad hoc rather than score-driven dynamics. In a static framework, Hafner et al. (2019) impose a multiplicative structure for the correlation matrix, which becomes additive under the matrix log transformation, and hence easier to estimate using minimum distance type estimators.

There is of course an identification issue because for an $(N \times N)$ correlation matrix, only $N(N-1) / 2$ elements are freely determinable. Consequently, for the matrix log of a correlation matrix, which is symmetric by definition, the diagonal elements are implied by the off-diagonal elements, although no analytical form is available in higher dimensions. Archakova and Hansen (2018) propose an algorithm that reconstructs the original correlation matrix based on the lower-triangular part, excluding the diagonal, of the log correlation matrix. We follow the same approach here by endowing this $N(N-1) / 2$ dimensional parameter with score-driven dynamics.

We propose two approaches for estimation, the first is a one-step estimator where all parts of the model are estimated simultaneously, which ensures statistical efficiency but may not be computationally feasible in high dimensions. In this case we assume equality of the conditional marginal distributions, so that the conditional distribution is indeed a multivariate student-t with only one degree-of-freedom parameter. The second is a twostep estimator where the volatilities are estimated in a first step, and the correlations in a second step. The marginal student-t distributions are allowed to have different degrees-offreedom parameters. This estimator, although statistically inefficient, is computationally 
simple and feasible even in higher dimensions. We derive the asymptotic estimation theory for both estimators.

In an empirical application to a set of six financial, commodity and cryptocurrency indices, we show that the proposed model fits well the data using two-step estimation and a variety of diagnostics and specification tests. We conclude that parameter restrictions across the components of the log correlation parameter may be employed that reduce the dimension of the parameter space and that are supported by classical information criteria.

The remainder of the paper is organized as follows. The following section recalls the definition and the structure of the matrix log of a correlation matrix. Section 3 introduces the model and proposes a one-step and two-step maximum likelihood estimator, including their corresponding asymptotic estimation theories. Section 4 presents a detailed empirical application of the model, and Section 5 concludes. Proofs of the theorems are collected in Appendix A. Appendix B shows that our model reduces to that of Harvey (2013), Section 7.3.1, in the bivariate Gaussian case, and tables and figures are collected in Appendix C.

\section{Matrix Logarithm of a Correlation matrix}

The matrix logarithm of a correlation matrix $\Theta$ is defined as $\log \Theta=\Gamma(\log \Lambda) \Gamma^{\top}$, where $\Gamma$ is the matrix containing the eigenvectors of $\Theta$ in its columns, and $\Lambda$ is a diagonal matrix containing the corresponding eigenvalues on the diagonal, which are all strictly positive. The diagonal matrix $\log \Lambda$ replaces the eigenvalues on the diagonal by their logarithm. The matrix $\log \Theta$ has a complicated structure, its diagonal elements can take any nonpositive values (see Lemma 2 of Archakova and Hansen 2018) and its off-diagonals can take any values. As an illustration, suppose that

$$
\Theta=\left(\begin{array}{ccc}
1 & 0.8 & 0.5 \\
0.8 & 1 & 0.2 \\
0.5 & 0.2 & 1
\end{array}\right), \text { then } \log \Theta=\left(\begin{array}{ccc}
-0.75 & 1.18 & 0.64 \\
1.18 & -0.55 & -0.07 \\
0.64 & -0.07 & -0.17
\end{array}\right)
$$


There are $N(N+1) / 2$ parameters in $\log \Theta$; we call these $\log$ parameters. On the other hand, $\Theta$ has only $N(N-1) / 2$ original parameters. For each $\Theta$, its $N(N-1) / 2$ original parameters completely identify its $N(N+1) / 2 \log$ parameters. In other words, there exists a function $f: \mathbb{R}^{N(N-1) / 2} \rightarrow \mathbb{R}^{N(N+1) / 2}$ which maps the original parameters to the log parameters. However, when $N>4, f$ does not have a closed form because when $N>4$ the continuous functions which map elements of a matrix to its eigenvalues have no closed form. When $N=2$, we can solve $f$ by hand (see Example 1 ).

Example 1. Suppose

$$
\Theta=\left(\begin{array}{ll}
1 & \rho \\
\rho & 1
\end{array}\right) .
$$

The eigenvalues of $\Theta$ are $1+\rho$ and $1-\rho$, respectively. The corresponding eigenvectors are $(1,1)^{\top} / \sqrt{2}$ and $(1,-1)^{\top} / \sqrt{2}$, respectively. Therefore

$$
\begin{aligned}
\log \Theta & =\left(\begin{array}{cc}
1 & 1 \\
1 & -1
\end{array}\right)\left(\begin{array}{cc}
\log (1+\rho) & 0 \\
0 & \log (1-\rho)
\end{array}\right)\left(\begin{array}{cc}
1 & 1 \\
1 & -1
\end{array}\right) \frac{1}{2} \\
& =\left(\begin{array}{cc}
\frac{1}{2} \log \left(1-\rho^{2}\right) & \frac{1}{2} \log \left(\frac{1+\rho}{1-\rho}\right) \\
\frac{1}{2} \log \left(\frac{1+\rho}{1-\rho}\right) & \frac{1}{2} \log \left(1-\rho^{2}\right)
\end{array}\right) .
\end{aligned}
$$

Thus

$$
f(\rho)=\left(\frac{1}{2} \log \left(1-\rho^{2}\right), \frac{1}{2} \log \left(\frac{1+\rho}{1-\rho}\right), \frac{1}{2} \log \left(1-\rho^{2}\right)\right)^{\top},
$$

and we see that $\rho$ generates two distinct entries for $\log \Theta$. The off-diagonal element $\frac{1}{2} \log \left(\frac{1+\rho}{1-\rho}\right)$ is the well-known Fisher's z-transformation of $\rho$. We also see that $\log \Theta$ is not only symmetric about the diagonal, but also symmetric about the cross-diagonal (from the upper right to the lower left).

The idea of Archakova and Hansen 2018 is to identify a subset of unrestricted log parameters and then fill in the remainder afterwards. They propose the following procedure. Let $\theta$ be the vector containing only the lower triangular portion of $\log \Theta$, excluding the 
diagonal. Then, the diagonal elements of $\log \Theta$ are uniquely determined by some function $\phi: \mathbb{R}^{N(N-1) / 2} \rightarrow \mathbb{R}^{N}$, which can be obtained numerically (in the case $N=2$ there is a closed form, see Example 1). Archakova and Hansen (2018) gave a concrete algorithm to do this and established its validity. We will use this idea in our model, starting from a dynamic parameter $\theta_{t}$, to first reconstruct the full $\log$ correlation matrix $\log \Theta_{t}$, and then by matrix exponentiation the original correlation matrix $\Theta_{t}$.

\section{The model and its estimation}

We introduce a model that combines conditional heteroskedasticity, dynamic conditional correlations, and conditional leptokurtosis in a dynamic score-driven framework. Consider the return vector for $N$ assets, $y_{t}=\left(y_{1 t}, \ldots, y_{N t}\right)^{\top}$. To simplify the presentation and discussion of the model, we will first assume that the conditional distribution of $y_{t}$ given the past is a multivariate student-t distribution, but later allow for marginal distributions with different degrees of freedom parameters.

We further assume that the parameter vector $\vartheta$ can be separated into a vector $\tau$ that characterizes the marginal distributions and the volatility part, a vector $\delta$ which characterizes the correlation part, and the degrees-of-freedom parameter $\eta$, and hence, $\vartheta=\left(\tau^{\top}, \delta^{\top}, \eta\right)^{\top}$.

The core of the model is the process generating the conditional correlation matrix $\Theta_{t}$. Before presenting this part of the model, we introduce the model framework for $y_{t}$ including the marginal dynamics and the distribution conditional on the information set $\mathcal{F}_{t-1}$,

$$
\begin{aligned}
y_{t} \mid \mathcal{F}_{t-1} & \sim F_{\eta, H_{t}}, \quad \eta>2 \\
H_{t}(\vartheta) & =D_{t}(\tau) \Theta_{t}(\delta) D_{t}(\tau) \\
D_{t}(\tau) & =\operatorname{diag}\left(\exp \left(h_{1 t}\right), \ldots, \exp \left(h_{N t}\right)\right)^{\top} \\
h_{i t}\left(\tau_{i}\right) & =\omega_{i}^{v}+\phi_{i}^{v} h_{i, t-1}+\kappa_{i}^{v} u_{i, t-1}^{v}, \quad i=1, \ldots, N \\
u_{i t}^{v} & =\frac{(\eta+1) y_{i t}^{2}}{(\eta-2) \exp \left(2 h_{t-1}\right)+y_{i t}^{2}}-1, \quad i=1, \ldots, N
\end{aligned}
$$


where $F_{\eta, H_{t}}$ is the standardized multivariate student-t distribution with $\eta$ d.o.f., $\eta>2$, and dispersion matrix $H_{t}$, i.e. its log density is given by

$$
f_{\eta, H_{t}}\left(y_{t}\right)=k(\eta)-\frac{1}{2}\left\{\log \left|H_{t}\right|+(\eta+N) \log \left(1+\frac{1}{\eta-2} y_{t}^{\top} H_{t}^{-1} y_{t}\right)\right\} .
$$

where $k(\eta)=\log \Gamma\left(\frac{\eta+N}{2}\right)-\log \Gamma\left(\frac{\eta}{2}\right)-\frac{N}{2} \log ((\eta-2) \pi)$. The structure of the model implies that the conditional mean of $y_{t}$ is zero, the conditional variances are given by $\exp \left(2 h_{i t}\right)$, and the conditional correlation matrix is given by $\Theta_{t}$. Each one of $D_{t}, \Theta_{t}$ and $H_{t}$ are assumed to be measurable functions of the information set $\mathcal{F}_{t-1}$.

Each asset's volatility is assumed to follow a univariate Beta-t-EGARCH process, where the score with respect to log volatility is given by (5). The only difference with respect to the standard formulation of this model in Harvey (2013) is that we do not consider the classical but the standardized student-t distribution, but the score is the same up to a scaling factor that depends on the degrees-of-freedom. It is straightforward to generalize this model to allow for volatility spillover, perhaps following some test for Granger causality in volatility. To keep the notation simple and estimation feasible in high dimensions, we refrain from doing so here as it does not affect our main interest, the modelling of the correlation part.

We denote by $\varepsilon_{t}=\left(\varepsilon_{1 t}, \ldots, \varepsilon_{N t}\right)^{\top}$ the vector of standardized residuals obtained after estimation of the volatility model, i.e., $\varepsilon_{t}=D_{t}(\tau)^{-1} y_{t}$, so that the components of $\varepsilon_{t}$ have conditional mean zero and conditional variance unity. However, the components of $\varepsilon_{t}$ are conditionally correlated, and the conditional correlation matrix is given by $\Theta_{t}=$ $\mathrm{E}\left[\varepsilon_{t} \varepsilon_{t}^{\top} \mid \mathcal{F}_{t-1}\right]$.

Now consider the following score-driven model for the conditional correlation matrix $\Theta_{t}(\delta)$. As in Archakova and Hansen (2018) we model the lower triangular part of $\log \Theta_{t}(\delta)$, excluding the diagonal, i.e., $\theta_{t}(\delta):=\operatorname{vecl}\left(\log \Theta_{t}(\delta)\right)$, and then fill in the diagonal elements to ensure that $\Theta_{t}$ is a correlation matrix. The model is a first order dynamic conditional score model for the log correlation parameter, i.e.

$$
\theta_{t}(\delta)=\omega+\Phi \theta_{t-1}(\delta)+K u_{t-1}
$$


where $u_{t}$ is the score of the likelihood function with respect to $\theta_{t}$, and $\Phi=\operatorname{diag}\left(\phi_{1}, \ldots, \phi_{N^{*}}\right)$ and $K=\operatorname{diag}\left(\kappa_{1}, \ldots, \kappa_{N^{*}}\right)$ are $N^{*} \times N^{*}$ diagonal parameter matrices, where $N^{*}=$ $N(N-1) / 2$. We restrict $\Phi$ and $K$ to be diagonal, as otherwise the number of parameters would be of order $O\left(N^{4}\right)$, which is infeasible in high dimensions. However, similar to the volatility case, one might consider "log correlation spillover", and tests thereof, but interpretation is not obvious since the link between the log parameters and correlations is not trivial.

The specific form of the score vector $u_{t}$ depends of course on the conditional distribution, which will be discussed in detail in the following in the context of maximum likelihood estimation. We distinguish between a multivariate student-t distribution, which is used for one-step estimation, and a Meta-t distribution which is used for two-step estimation. The score vectors in both cases are very similar but not exactly the same. Furthermore, one may use standardized versions of the score vector, by pre-multiplying $u_{t}$ by the inverse of the (square root of the) information matrix, see e.g. the model of Harvey 2013, Section 7.3.1., which is motivated in particular for cases where the standardized score is an i.i.d. random vector. In our case, however, this will not be the case in general, so to keep things simple we will not pursue this strategy here.

Although one of the main advantages of our model specification lies in the separation of volatility and correlation parts, just as in Engle (2002) and related works, it would be more efficient from a statistical viewpoint to do the estimation of all model parameters in one step. In the following, we therefore present both approaches, one-step and two-step estimation.

\subsection{One step estimation}

For one-step estimation we assume for simplicity that $\eta_{1}=\ldots=\eta_{N}=\eta$, so that the conditional joint distribution of $y_{t}$ is a multivariate student-t. The log likelihood function for a sample of $T$ observations as a function of the parameter vector $\vartheta=\left(\tau^{\top}, \delta^{\top}, \eta\right)^{\top}$, 
$\delta:=\left(\omega^{\top}, \operatorname{diag}(\Phi)^{\top}, \operatorname{diag}(K)^{\top}\right)^{\top}$ takes the form

$$
\begin{aligned}
L(\vartheta)=\sum_{t=1}^{T} l_{t}(\vartheta)= & T \log \Gamma\left(\frac{\eta+N}{2}\right)-T \log \Gamma\left(\frac{\eta}{2}\right)-\frac{T N}{2} \log ((\eta-2) \pi) \\
& -\frac{1}{2} \sum_{t=1}^{T}\left\{2 \log \left|D_{t}(\tau)\right|+\log \left|\Theta_{t}(\delta)\right|+(\eta+N) \log \left(1+\frac{1}{\eta-2} \varepsilon_{t}^{\top} \Theta_{t}(\delta)^{-1} \varepsilon_{t}\right)\right\}
\end{aligned}
$$

The spectral decomposition of the log correlation matrix is denoted by $\log \Theta_{t}=\Gamma_{t} \Lambda_{t} \Gamma_{t}^{\top}$, where $\Gamma_{t}$ is the $(N \times N)$ matrix whose columns are the eigenvectors of $\log \Theta$, and $\Lambda_{t}$ is the $(N \times N)$ diagonal matrix containing the corresponding eigenvalues $\lambda_{i t}, i=1, \ldots, N$ on the diagonal. Define the matrix $A_{t}:=\left(\Gamma_{t} \otimes \Gamma_{t}\right) \Xi_{t}\left(\Gamma_{t} \otimes \Gamma_{t}\right)^{\top}$, where $\Xi_{t}$ is the $N^{2} \times N^{2}$ matrix with elements given by

$$
\xi_{i j}=\Xi_{(i-1) N+j,(i-1) N+j}= \begin{cases}\exp \left(\lambda_{i t}\right), & \text { if } \lambda_{i t}=\lambda_{j t} \\ \frac{\exp \left(\lambda_{i t}\right)-\exp \left(\lambda_{j t}\right)}{\lambda_{i t}-\lambda_{j t}}, & \text { if } \lambda_{i t} \neq \lambda_{j t}\end{cases}
$$

for $i, j=1, \ldots, N$. Furthermore, define the following elimination matrices: $E_{d}\left(N \times N^{2}\right)$, $E_{l}\left(N(N-1) / 2 \times N^{2}\right)$, and $E_{u}\left(N(N-1) / 2 \times N^{2}\right)$ such that $\operatorname{vecl}(Q)=E_{l} \operatorname{vec}(Q)$, $\operatorname{vecl}\left(Q^{\top}\right)=E_{u} \operatorname{vec}(Q)$ and $\operatorname{diag}(Q)=E_{d} \operatorname{vec}(Q)$ for any square matrix $Q$, and $E_{s}:=$ $E_{l}+E_{u}$. Finally, define the permutation matrix $P$ as $P\left(\operatorname{diag}(Q)^{\top}, \operatorname{vecl}(Q)^{\top}\right)^{\top}=\operatorname{vec}(Q)$ for any symmetric conformable matrix $Q$. With this notation, the following theorem provides the analytic form of the score with respect to the log correlation parameter.

Theorem 1. The score with respect to the log correlation parameter $\theta_{t}$ is given by

$$
u_{t}=\frac{\partial l_{t}}{\partial \theta_{t}}=\frac{1}{2} M_{t} v e c\left(\frac{\eta+N}{\eta-2+\varepsilon_{t}^{\top} \Theta_{t}^{-1} \varepsilon_{t}} \Theta_{t}^{-1} \varepsilon_{t} \varepsilon_{t}^{\top} \Theta_{t}^{-1}-\Theta_{t}^{-1}\right)
$$

where

$$
M_{t}:=\left(\begin{array}{c}
-\left(E_{d} A_{t} E_{d}^{\top}\right)^{-1} E_{d} A_{t} E_{s}^{\top} \\
I_{N(N-1) / 2}
\end{array}\right)^{\top} P^{\top} A_{t}
$$

Remark 1. In the limiting case $\eta \rightarrow \infty$, we obtain the Gaussian distribution as a special case, for which we let $\vartheta=\left(\tau^{\top}, \delta^{\top}\right)^{\top}$, and the log likelihood function takes the form

$$
L(\vartheta)=-\frac{T N}{2} \log (2 \pi)-\frac{1}{2} \sum_{t=1}^{T}\left\{2 \log \left|D_{t}(\tau)\right|+\log \left|\Theta_{t}(\delta)\right|+\varepsilon_{t}^{\top} \Theta_{t}^{-1}(\delta) \varepsilon_{t}\right\}
$$


and the score reduces to

$$
u_{t}=\frac{\partial l_{t}}{\partial \theta_{t}}=\frac{1}{2}\left(\begin{array}{c}
-\left(E_{d} A_{t} E_{d}^{\top}\right)^{-1} E_{d} A_{t} E_{s}^{\top} \\
I_{N(N-1) / 2}
\end{array}\right)^{\top} P^{\top} A_{t} v e c\left(\Theta_{t}^{-1} \varepsilon_{t} \varepsilon_{t}^{\top} \Theta_{t}^{-1}-\Theta_{t}^{-1}\right)
$$

Remark 2. Note that the score can be written as $u_{t}=g\left(\theta_{t}\right) Z_{t}$ where $g\left(\theta_{t}\right)=\frac{1}{2} M_{t}\left(\Theta_{t}^{-1 / 2} \otimes\right.$ $\left.\Theta_{t}^{-1 / 2}\right)$, and $Z_{t}=\operatorname{vec}\left(\frac{\eta+N}{\eta-2+\xi_{t}^{\top} \xi_{t}} \xi_{t} \xi_{t}^{\top}-I_{N}\right)$ is an i.i.d. random vector. The score vector $u_{t}$ is a martingale difference sequence, which can be shown using results of Fiorentini et al. 2003. However, it is not i.i.d. in general, although it is i.i.d. in the univariate case because, in that case, $g\left(\theta_{t}\right)=1 / 2$.

Remark 3. The process $\theta_{t}$ has an SRE representation as

$$
\theta_{t}=\psi_{t}\left(\theta_{t-1}\right)
$$

where $\psi_{t}(s)=\omega+\phi s+\kappa g(s) Z_{t}$, which can be shown to be a Lipschitz map with maximum Lipschitz coefficient $\Lambda(\psi)<\infty$.

The volatility part of the likelihood, i.e., $D_{t}(\tau)$ in $(7)$, is straightforward to calculate and exactly the same as in univariate Beta-t-EGARCH models. For the correlation part we use the following algorithm to compute $\Theta_{t}(\delta)$ and the score $u_{t}$ :

1. Initialization: Set $u_{0}=0$ and $\theta_{0}=\left(I_{N(N-1) / 2}-\Phi\right)^{-1} \omega$.

2. For $t=1, \ldots, T$ :

(a) Calculate $\theta_{t}$ as a function of past observations according to (6).

(b) Reconstruct $\operatorname{diag}\left(\log \Theta_{t}\right)$ using the algorithm of Archakova and Hansen (2018).

(c) Calculate the matrix exponential of $\log \Theta_{t}$ to obtain $\Theta_{t}$.

(d) Calculate the score $u_{t}$ according to (8).

3. Calculate the log likelihood (7). 
The MLE is then defined as the maximizer of the log likelihood function over some parameter space $\Omega$,

$$
\hat{\vartheta}=\arg \max _{\vartheta \in \Omega} L(\vartheta)
$$

No analytical solution is available, but standard numerical optimization routines can be employed. For the estimation theory we use the following assumptions.

(A1) The conditional distribution of $y_{t}$ is a standardized multivariate student-t distribution with $\eta$ degrees of freedom, $\eta>2$ and dispersion matrix $H_{t}$.

(A2) The parameter vector $\vartheta \in \Omega$, where $\Omega$ is a compact subset of $\mathbb{R}^{J}, J=\operatorname{dim}(\vartheta)$, such that $\left|\phi_{i}^{v}\right|<1, i=1 \ldots, N,\left|\phi_{j}\right|<1, j=1 \ldots, N^{*}, \kappa_{i}^{v} \neq 0, i=1, \ldots, N$, and $\kappa_{j} \neq 0, j=1, \ldots, N^{*}$.

(A3) The true parameter $\vartheta_{0}$ lies in the interior of $\Omega$.

$\left|\left(\phi_{i}^{v}\right)^{2}+2 \kappa_{i}^{v} \phi_{i}^{v} \mathrm{E}\left(\partial u_{i t}^{v} / \partial h_{i t}\right)\right|+\left(\kappa_{i}^{v}\right)^{2} \mathrm{E}\left[\left(\partial u_{i t}^{v} / \partial h_{i t}\right)^{2}\right]<1, \quad i=1, \ldots, N$

(A6) $\mathrm{E}\left[\log ^{+}\left(\Lambda\left(\psi_{t}\right)\right)<\infty\right.$ and $\mathrm{E}\left[\log \left(\Lambda\left(\psi_{t}\right)\right)<0\right.$.

Assumption (A2) is standard and necessary for identifiability and stationarity of the volatility and log correlation processes. (A3) is also standard and is needed for asymptotic normality. (A4) and (A5) ensure that the scores with respect to log volatility and log correlation, respectively, and their first derivatives, have finite second moments. They are also standard in this literature, see e.g. the conditions of Theorem 1 of Harvey (2013). Assumption (A6) is a contraction condition which ensures that the process $\theta_{t}$ is a stationary and ergodic sequence.

Let $\vartheta=\left(\tau^{\top}, \delta^{\top}, \eta\right)^{\top}$, and decompose the information matrix as

$$
I=\left(\begin{array}{ccc}
I_{\tau \tau} & I_{\tau \delta} & I_{\tau \eta} \\
I_{\tau \delta}^{\top} & I_{\delta \delta} & I_{\delta \eta} \\
I_{\tau \eta}^{\top} & I_{\delta \eta}^{\top} & I_{\eta \eta}
\end{array}\right)
$$

We then have the following result. 
Theorem 2. Under Assumptions (A1)-(A5), $\hat{\vartheta} \rightarrow_{p} \vartheta$ and the asymptotic distribution of the estimator in (13) is given by

$$
\sqrt{T}(\hat{\vartheta}-\vartheta) \stackrel{\mathcal{L}}{\rightarrow} N\left(0, I^{-1}\right)
$$

where $I$ is given in (14) with

$$
\begin{aligned}
I_{\delta \delta} & =\mathbb{E}\left[\frac{\partial \theta_{t}^{T}}{\partial \delta} M_{t}\left\{\frac{N+\eta}{2(N+\eta+2)}\left(\Theta_{t}^{-1} \otimes \Theta_{t}^{-1}\right)-\frac{1}{2(N+\eta+2)} \operatorname{vec}\left(\Theta_{t}^{-1}\right) \operatorname{vec}\left(\Theta_{t}^{-1}\right)^{\top}\right\} M_{t}^{\top} \frac{\partial \theta_{t}}{\partial \delta^{T}}\right] \\
I_{\eta \eta} & =\frac{\eta^{4}}{4}\left[\psi^{\prime}\left(\frac{\eta}{2}\right)-\psi^{\prime}\left(\frac{N+\eta}{2}\right)\right]-\frac{N \eta^{4}\left[\eta^{2}+N(\eta-4)-8\right]}{2(\eta-2)^{2}(N+\eta)(N+\eta+2)} \\
I_{\delta \eta} & =-\frac{N+2) \eta^{2}}{(\eta-2)(N+\eta)(N+\eta+2)} \mathbb{E} \frac{\partial \theta_{t}^{\top}}{\partial \delta} M_{t} \operatorname{vec}\left(\Theta_{t}^{-1}\right) \\
I_{\tau \tau} & =\mathbb{E}\left[\frac{\partial h_{t}^{\top}}{\partial \tau}\left\{\frac{N+\eta}{2(N+\eta+2)}\left(H_{t}^{-1} \otimes H_{t}^{-1}\right)-\frac{1}{2(N+\eta+2)} \operatorname{vec}\left(H_{t}^{-1}\right) \operatorname{vec}\left(H_{t}^{-1}\right)^{\top}\right\} \frac{\partial h_{t}}{\left.\partial \tau^{\top}\right]}\right. \\
I_{\tau \delta} & =\mathbb{E}\left[\frac{\partial h_{t}^{\top}}{\partial \tau}\left\{\frac{N+\eta}{2(N+\eta+2)}\left(H_{t}^{-1} \otimes H_{t}^{-1}\right)-\frac{1}{2(N+\eta+2)} \operatorname{vec}\left(H_{t}^{-1}\right) \operatorname{vec}\left(H_{t}^{-1}\right)^{\top}\right\}\left(D_{t} \otimes D_{t}\right) M_{t}^{\top} \frac{\partial \theta_{t}}{\partial \delta^{\top}}\right] \\
I_{\tau \eta} & =-\frac{N+2) \eta^{2}}{(\eta-2)(N+\eta)(N+\eta+2)} \mathbb{E} \frac{\partial v e c^{\top}\left(H_{t}\right)}{\partial \tau} \operatorname{vec}\left(H_{t}^{-1}\right)
\end{aligned}
$$

The information matrix further depends on the derivative of the log correlation parameter $\theta_{t}$ with respect to $\delta$, which is given by

$$
\frac{\partial \theta_{t}}{\partial \delta^{\top}}=z_{t-1}+x_{t-1} \frac{\partial \theta_{t-1}}{\partial \delta^{\top}}
$$

where

$$
z_{t}=\left(\begin{array}{lll}
I_{N^{*}} & \operatorname{diag}\left(\theta_{1 t}, \ldots, \theta_{N^{*} t}\right) & \operatorname{diag}\left(u_{1 t}, \ldots, u_{N^{*} t}\right)
\end{array}\right), \quad x_{t}=\Phi+K \frac{\partial u_{t}}{\partial \theta_{t}^{\top}}
$$

The information matrix can be estimated consistently by evaluating it at $\hat{\vartheta}$ and replacing the expectation operators by sample averages.

\subsection{Two-step estimation}

For two-step estimation we allow for different d.o.f. parameters of the marginal distributions, $\eta_{1}, \ldots, \eta_{N}$. Thus we now assume a conditional Meta-t distribution with degrees 
of freedom parameter $\eta$ and scale matrix $\Theta_{t}$, meaning that the conditional dependence structure of $y_{t}$ is determined by a student-t copula with scale matrix $\Theta_{t}$, see e.g. Demarta and McNeil (2005). for details on Meta-t distributions and the student-t copula.

As before, the conditional marginal distributions are given by score-driven volatility processes and student-t distributions, but now with potentially different degrees of freedom $\eta_{i}$. If all d.o.f. parameters happen to be the same and equal to $\eta$, then this model reduces to the one considered for one-step estimation, and in this case two-step estimation would be less efficient than one-step estimation, although it might be the only feasible estimation procedure computationally, at least in high dimensions.

Formally, for the theory we replace Assumption (A1) by the following.

(A1') The conditional distribution of $y_{t}$ is given by

$$
F_{t}\left(y_{1 t}, \ldots, y_{N t}\right)=C_{\eta, \Theta t}\left(F_{\eta_{1}, h_{1 t}}\left(y_{1 t}\right), \ldots, F_{\eta_{N}, h_{N t}}\left(y_{N t}\right)\right)
$$

with $\eta>2$ and $\eta_{i}>2, i=1 \ldots, N$, where $F_{\eta_{i}, h_{i t}}$ are the standardized student-t marginal cdf's with $\log$ scale $h_{i t}$, and $C_{\eta, \Theta_{t}}$ is the cdf of a standardized student-t copula with dispersion matrix $\Theta_{t}$.

Note that the copula is invariant to standardizations of the marginal distributions, so that the student-t copula with scale matrix $H_{t}$ is the same as the student-t copula with scale matrix $\Theta_{t}$. Note also that if all marginal degrees of freedom are the same and equal to the copula parameter $\eta$, then Assumption (A1') collapses to (A1).

We augment the vector of parameters characterizing the marginal distributions as $\tau_{i}=\left(\omega_{i}^{v}, \phi_{i}^{v}, \kappa_{i}^{v}, \eta_{i}\right)^{\top}, i=1, \ldots, N$, and $\tau=\left(\tau_{1}^{\top}, \ldots, \tau_{N}^{\top}\right)^{\top}$. The copula density has the form

$$
c_{\eta, \Theta_{t}}(u)=\frac{f_{\eta, \Theta_{t}}\left(t_{\eta}^{-1}\left(u_{1}\right), \ldots, t_{\eta}^{-1}\left(u_{N}\right)\right)}{\prod_{i=1}^{N} g_{\eta}\left(t_{\eta}^{-1}\left(u_{i}\right)\right)}
$$

see e.g. Demarta and McNeil (2005), where $f_{\eta, \Theta_{t}}(\cdot)$ is the joint pdf of a multivariate standardized student-t distribution with scale matrix $\Theta_{t}$, and $t_{\eta}(\cdot)$ and $g_{\eta}(\cdot)$ are standardized univariate student-t cdf and pdf, respectively. The conditional joint density of $y_{t}$ is then 
given by

$$
f_{t}\left(y_{t}\right)=c_{\eta, \Theta}\left(F_{\eta_{1}, h_{1 t}}\left(y_{1 t}\right), \ldots, F_{\eta_{N}, h_{N t}}\left(y_{N t}\right)\right) \prod_{i=1}^{N} g_{\eta_{i}, h_{i t}}\left(y_{i t}\right)
$$

which is used to construct the likelihood function.

The log likelihood function for a sample of $T$ observations as a function of the parameter vector $\vartheta=\left(\tau^{\top}, \delta^{\top}, \eta\right)^{\top}, \delta:=\left(\omega^{\top}, \phi^{\top}, \kappa^{\top}\right)^{\top}$ takes the form

$$
\begin{aligned}
L(\vartheta) & =L^{V}(\tau)+L^{C}(\vartheta) \\
L^{V}(\tau) & =\sum_{i=1}^{N} l_{i}^{V}(\tau) \\
l_{i}^{V}(\tau) & =c\left(\eta_{i}, 1\right)-\frac{1}{2} \sum_{t=1}^{T}\left\{2 h_{i t}+\left(\eta_{i}+1\right) \log \left(1+\frac{y_{i t}^{2} \exp \left(-2 h_{i t}\right)}{\eta_{i}-2}\right)\right\} \\
L^{C}(\vartheta) & =c(\eta, N)-N c(\eta, 1)-\frac{1}{2} \sum_{t=1}^{T} l_{t}^{C}(\vartheta) \\
l_{t}^{C}(\vartheta) & =\log \left|\tilde{\Theta}_{t}(\delta)\right|+(\eta+N) \log \left(1+\frac{{\tilde{\varepsilon_{t}}}^{\top} \tilde{\Theta}_{t}(\delta)^{-1}{\tilde{\varepsilon_{t}}}^{\eta}}{\eta-2}\right)-\sum_{i=1}^{N}(\eta+1) \log \left(1+\frac{{\tilde{\varepsilon_{i t}}}^{2}}{\eta-2}\right) \\
c(\eta, N) & =T \log \Gamma\left(\frac{\eta+N}{2}\right)-T \log \Gamma\left(\frac{\eta}{2}\right)-\frac{T N}{2} \log ((\eta-2) \pi)
\end{aligned}
$$

where $\tilde{\varepsilon}_{i t}:=s_{\eta}\left(\varepsilon_{i t}, \eta_{i}\right)=t_{\eta}^{-1}\left(t_{\eta_{i}}\left(\varepsilon_{i t}\right)\right)$ is a mapping of the quantiles of a standardized student-t distribution with $\eta_{i}$ d.o.f. into the quantiles of a standardized student-t with $\eta$ d.o.f. The mapping function $s_{\eta}(\cdot)$ is strictly monotone and its inverse is given by $s_{\eta}^{-1}\left(\cdot, \eta_{i}\right)=t_{\eta_{i}}^{-1}\left(t_{\eta}(\cdot)\right)$. When applied to a vector, we define $s_{\eta}(\cdot)$ as $s_{\eta}\left(\varepsilon_{t}, \underline{\eta}\right)=$ $\left(s_{\eta}\left(\varepsilon_{1 t}, \eta_{1}\right), \ldots, s_{\eta}\left(\varepsilon_{N t}, \eta_{N}\right)\right)^{\top}$, where $\underline{\eta}=\left(\eta_{1}, \ldots, \eta_{N}\right)^{\top}$, and similarly for $s_{\eta}^{-1}\left(\varepsilon_{t}, \underline{\eta}\right)$.

Note that the likelihood reduces to that of the one-step estimation when imposing the restriction $\eta_{1}=\ldots=\eta_{N}=\eta$.

The matrix $\tilde{\Theta}_{t}(\delta)$ is such that $\operatorname{vecl}\left(\log \tilde{\Theta}_{t}(\delta)\right)=: \tilde{\theta}_{t}(\delta)$ follows the same process $(6)$ as above for the one-step method, but in the score $u_{t}$ replacing $\Theta_{t}(\delta)$ by $\tilde{\Theta}_{t}(\delta)$ and $\varepsilon_{t}$ by $\tilde{\varepsilon}_{t}$, i.e.

$$
\begin{aligned}
\tilde{\theta}_{t}(\delta) & =\omega+\Phi \tilde{\theta}_{t-1}(\delta)+K \tilde{u}_{t-1} \\
\tilde{u}_{t} & =\frac{1}{2} \tilde{M}_{t} \operatorname{vec}\left(\frac{\eta+N}{\eta-2+\tilde{\varepsilon}_{t}^{\top} \tilde{\Theta}_{t}^{-1} \tilde{\varepsilon}_{t}} \tilde{\Theta}_{t}^{-1} \tilde{\varepsilon}_{t} \tilde{\varepsilon}_{t}^{\top} \tilde{\Theta}_{t}^{-1}-\tilde{\Theta}_{t}^{-1}\right)
\end{aligned}
$$


and where $\tilde{M}_{t}$ is constructed as $M_{t}$ above but replacing $\Theta_{t}$ by $\tilde{\Theta}_{t}(\delta)$.

Now, due to the nonlinear transformation $s_{\eta}(\cdot), \tilde{\Theta}_{t}(\delta)$ is not exactly equal to the conditional correlation matrix $\Theta_{t}(\delta)$. It can however be reconstructed easily by numerical methods. For example, for a given $\tilde{\Theta}_{t}$, generate a large number $K$ of r.v. $X$ with standardized multivariate student-t distribution with $\eta$ d.o.f. and correlation matrix $\tilde{\Theta}_{t}$. Then, obtain the sample correlation matrix of the transformed data as

$$
\Theta_{t}=\frac{1}{K} \sum_{k=1}^{K} s_{\eta}^{-1}\left(X_{k}, \underline{\eta}\right)\left(s_{\eta}^{-1}\left(X_{k}, \underline{\eta}\right)\right)^{\top}
$$

The diagonal elements of $\Theta_{t}$ should be very close to one if $K$ is sufficiently large. To illustrate this mapping of correlations, we plot in Figure 1 the correlation $\rho$ as a function of $\tilde{\rho}$ for the case $\eta=7, \eta_{1}=4$ and $\eta_{2}=10$, obtained by simulating $K=10^{7}$ bivariate student-t random variables. If all degrees-of-freedom parameters were the same, then this would be the identity function. Here this function is different, but very close to the identity function, with the difference shown on the right of the Figure. Hence, after transformation the correlations change but only slightly so.

The sum of the marginal log likelihood functions, $L^{V}(\tau)$, only depends on the marginal parameters, not on the copula parameters. It is in fact equal to the sum of the univariate volatility log likelihoods that are equal to the Beta-t-EGARCH model log likelihoods. In the first step, the inference function for margins approach proposed by Joe (1997) maximizes $L^{V}(\tau)$ with respect to $\tau$ to obtain $\hat{\tau}$, which is then used in a second step to maximize $L^{C}\left(\hat{\tau}^{\top}, \delta, \eta\right)^{\top}$ with respect to $\delta$ and $\eta$, to give $\hat{\delta}$ and $\hat{\eta}$. Then, $\hat{\vartheta}=\left(\hat{\tau}^{\top}, \hat{d}^{\top}\right)^{\top}$, where $d=\left(\delta^{\top}, \eta\right)^{\top}$ is the parameter characterizing the copula, and $\hat{d}=\left(\hat{\delta}^{\top}, \hat{\eta}\right)^{\top}$.

We now have the following result.

Theorem 3. Under Assumptions (A1')-(A5), $\hat{\vartheta} \rightarrow_{p} \vartheta$ and

$$
\sqrt{T}(\hat{\vartheta}-\vartheta) \rightarrow_{d} N\left(0,(-F)^{-1} G(-F)^{-1}\right)
$$



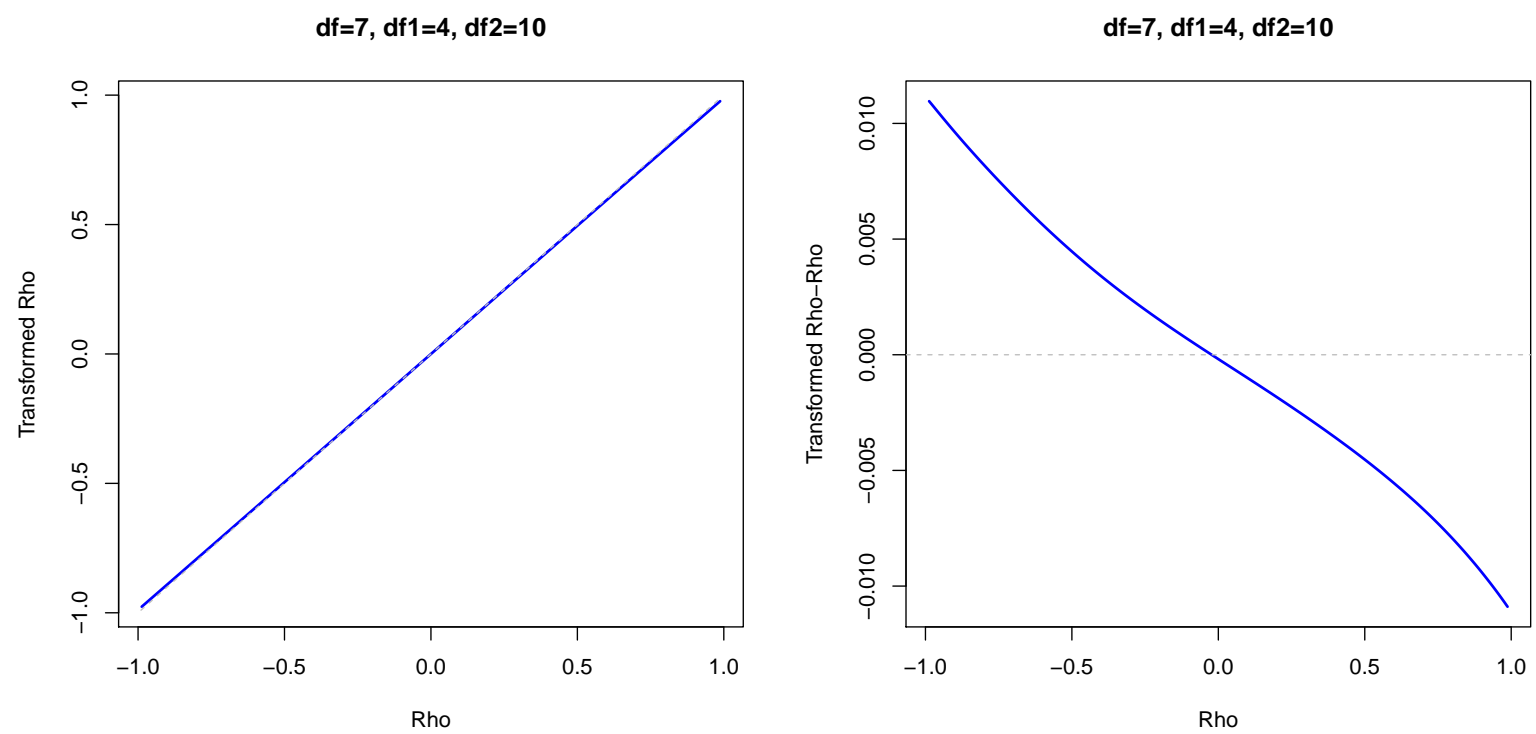

Figure 1: The left panel shows the correlation $\rho$ as a function of $\tilde{\rho}$, where $\tilde{\rho}$ is the correlation parameter of a bivariate standardized student-t r.v. $X$ with $\eta=7$ d.o.f., and $\rho$ is the correlation of transformed r.v. $s_{\eta}^{-1}\left(X_{1}, \eta_{1}\right)$ and $s_{\eta}^{-1}\left(X_{2}, \eta_{2}\right)$ with $\eta_{1}=4$ and $\eta_{2}=10$. The right panel shows the difference of this function from the identity function.

where

$$
-F=\left(\begin{array}{cccc}
\mathcal{J}_{11} & \cdots & 0 & 0 \\
\vdots & \ddots & \vdots & \vdots \\
0 & \cdots & \mathcal{J}_{N N} & 0 \\
\mathcal{I}_{d 1} & \cdots & \mathcal{I}_{d N} & \mathcal{I}_{d d}
\end{array}\right), \quad G=\left(\begin{array}{cccc}
\mathcal{J}_{11} & \cdots & \mathcal{J}_{1 N} & 0 \\
\vdots & \ddots & \vdots & \vdots \\
\mathcal{J}_{N 1} & \cdots & \mathcal{J}_{N N} & 0 \\
0 & \cdots & 0 & \mathcal{I}_{d d}
\end{array}\right)
$$

and where

$$
\begin{aligned}
& \mathcal{J}_{i j}=E\left[\frac{\partial l_{i}^{V}\left(\tau_{i}\right)}{\partial \tau_{j}} \frac{\partial l_{j}^{V}(\tau)}{\partial \tau^{\top}}\right], \quad i, j=1 \ldots, N \\
& \mathcal{I}_{d d}=-E\left[\frac{\partial^{2} l(\vartheta)}{\partial d \partial d^{\top}}\right], \\
& \mathcal{I}_{d i}=-E\left[\frac{\partial^{2} l(\vartheta)}{\partial d \partial \tau_{i}^{\top}}\right], \quad i=1 \ldots, N
\end{aligned}
$$

Note that the matrices $J_{i i}, i=1 \ldots, N$, are the information matrices of the marginal 
likelihoods, so well-known results of univariate Beta-t-EGARCH models can be used for their calculation, see e.g. Proposition 20 of Harvey (2013).

The components of $\mathcal{I}$ are given in the following. Note first that

$$
\begin{aligned}
\frac{\partial^{2} l_{t}^{C}}{\partial \delta \partial \tau_{i}^{\top}}=\frac{\partial \tilde{\theta}_{t}^{\top}}{\partial \delta} \frac{\partial \tilde{u}_{t}}{\partial \tau_{i}^{\top}}+\left(\tilde{u}_{t}^{\top} \otimes I_{m}\right) \frac{\partial \operatorname{vec}}{\partial \tau_{i}^{\top}} \frac{\partial \tilde{\theta}_{t}^{\top}}{\partial \delta} \\
\frac{\partial^{2} l_{t}^{C}}{\partial \delta \partial \delta^{\top}}=\frac{\partial \tilde{\theta}_{t}^{\top}}{\partial \delta} \frac{\partial \tilde{u}_{t}}{\partial \delta^{\top}}+\left(\tilde{u}_{t}^{\top} \otimes I_{m}\right) \frac{\partial \operatorname{vec}}{\partial \delta^{\top}} \frac{\partial \tilde{\theta}_{t}^{\top}}{\partial \delta},
\end{aligned}
$$

where $m=\operatorname{dim}(\delta)$ and $\partial \tilde{\theta}_{t}^{\top} / \partial \delta$ is given in (16), replacing $\theta_{t}$ by $\tilde{\theta}_{t}, u_{t}$ by $\tilde{u}_{t}, z_{t}$ by $\tilde{z}_{t}$ and $x_{t}$ by $\tilde{x}_{t}$. Furthermore,

$$
\begin{aligned}
& \frac{\partial \operatorname{vec}}{\partial \tau_{i}^{\top}} \frac{\partial \tilde{\theta}_{t}^{\top}}{\partial \delta}=\frac{\partial \operatorname{vec}\left(\tilde{z}_{t-1}^{\top}\right)}{\partial \tau_{i}^{\top}}+\left(\tilde{x}_{t-1} \otimes I_{m}\right) \frac{\partial \operatorname{vec}}{\partial \tau_{i}^{\top}} \frac{\partial \tilde{\theta}_{t-1}^{\top}}{\partial \delta}+\left(I_{N^{*}} \otimes \frac{\partial \tilde{\theta}_{t-1}^{\top}}{\partial \delta}\right) \frac{\partial \operatorname{vec}\left(\tilde{x}_{t-1}^{\top}\right)}{\partial \tau_{i}^{\top}} \\
& \frac{\partial \operatorname{vec}}{\partial \delta^{\top}} \frac{\partial \tilde{\theta}_{t}^{\top}}{\partial \delta}=\frac{\partial \operatorname{vec}\left(\tilde{z}_{t-1}^{\top}\right)}{\partial \delta^{\top}}+\left(\tilde{x}_{t-1} \otimes I_{m}\right) \frac{\partial \operatorname{vec}}{\partial \delta^{\top}} \frac{\partial \tilde{\theta}_{t-1}^{\top}}{\partial \delta}+\left(I_{N^{*}} \otimes \frac{\partial \tilde{\theta}_{t-1}^{\top}}{\partial \delta}\right) \frac{\partial \operatorname{vec}\left(\tilde{x}_{t-1}^{\top}\right)}{\partial \delta^{\top}}
\end{aligned}
$$

Next we calculate the derivatives involving the degrees-of-freedom parameter $\eta$. The first derivative is given by

$$
\begin{aligned}
\frac{\partial l}{\partial \eta} & =\frac{\partial c(\eta, N)}{\partial \eta}-N \frac{\partial c(\eta, 1)}{\partial \eta}-\frac{1}{2} \sum_{t=1}^{T} \frac{\partial l_{t}^{C}}{\partial \eta} \\
\frac{\partial l_{t}^{C}}{\partial \eta} & =\tilde{u}_{t}^{\top} \frac{\partial \tilde{\theta}_{t}^{\top}}{\partial \eta}+\log \left(1+\frac{\tilde{\varepsilon}_{t}^{\tau} \tilde{\Theta}_{t}^{-1} \tilde{\varepsilon}_{t}}{\eta-2}\right)+\frac{\eta+N}{\eta-2+\tilde{\varepsilon}_{t}^{\top} \tilde{\Theta}_{t}^{-1} \tilde{\varepsilon}_{t}}\left\{2 \tilde{\varepsilon}_{t}^{\top} \tilde{\Theta}_{t}^{-1} \frac{\partial \tilde{\varepsilon}_{t}}{\partial \eta}-\frac{\tilde{\varepsilon}_{t}^{t} \tilde{\Theta}_{t}^{-1} \tilde{\varepsilon}_{t}}{\eta-2}\right\} \\
& -\sum_{i=1}^{N} \log \left(1+\frac{\tilde{\varepsilon}_{i t}^{2}}{\eta-2}\right)+\frac{\eta+1}{\eta-2+\tilde{\varepsilon}_{i t}^{2}}\left\{2 \tilde{\varepsilon}_{i t} \frac{\partial \tilde{\varepsilon}_{i t}}{\partial \eta}-\frac{\tilde{\varepsilon}_{i t}^{2}}{\eta-2}\right\}
\end{aligned}
$$

The second derivatives can be obtained by straightforward calculations. First, we have

$$
\begin{aligned}
\frac{\partial^{2} l_{t}^{C}}{\partial \eta \partial \tau_{i}^{\top}} & =\frac{\partial \tilde{\theta}_{t}^{\top}}{\partial \eta} \frac{\partial \tilde{u}_{t}}{\partial \tau_{i}^{\top}}+\tilde{u}_{t}^{\top} \frac{\partial^{2} \tilde{\theta}_{t}}{\partial \eta \partial \tau_{i}^{\top}} \\
& +\left\{\frac{\eta+N}{\eta-2+\tilde{\varepsilon}_{t}^{\top} \tilde{\Theta}_{t}^{-1} \tilde{\varepsilon}_{t}}\left(2 \tilde{\varepsilon}_{t}^{\top} \tilde{\Theta}_{t}^{-1} \frac{\partial \tilde{\varepsilon}_{t}}{\partial \eta}-\frac{\tilde{\varepsilon}_{t}^{\top} \tilde{\Theta}_{t}^{-1} \tilde{\varepsilon}_{t}}{\eta-2}\right)-\frac{N+2}{\eta-2}\right\}\left(\eta-2+\tilde{\varepsilon}_{t}^{\top} \tilde{\Theta}_{t}^{-1} \tilde{\varepsilon}_{t}\right)^{-1} \frac{\partial \tilde{\varepsilon}_{t}^{\top} \tilde{\Theta}_{t}^{-1} \tilde{\varepsilon}_{t}}{\partial \tau_{i}^{\top}} \\
& -\left\{\frac{\eta+1}{\eta-2+\tilde{\varepsilon}_{i t}^{2}}\left(2 \tilde{\varepsilon}_{i t} \frac{\partial \tilde{\varepsilon}_{i t}}{\partial \eta}-\frac{\tilde{\varepsilon}_{i t}^{2}}{\eta-2}\right)-\frac{3}{\eta-2}\right\}\left(\eta-2+\tilde{\varepsilon}_{i t}^{2}\right)^{-1} 2 \tilde{\varepsilon}_{i t} \frac{\partial \tilde{\varepsilon}_{i t}}{\partial \tau_{i}^{\top}} \\
& +\frac{2(\eta+N)}{\eta-2+\tilde{\varepsilon}_{t}^{\top} \tilde{\Theta}_{t}^{-1} \tilde{\varepsilon}_{t}}\left(\tilde{\varepsilon}_{t}^{\top} \tilde{\Theta}_{t}^{-1} \frac{\partial^{2} \tilde{\varepsilon}_{t}}{\partial \eta \partial \tau_{i}^{\top}}+\frac{\partial \tilde{\varepsilon}_{t}^{\top}}{\partial \eta} \frac{\partial \tilde{\Theta}_{t}^{-1} \tilde{\varepsilon}_{t}}{\partial \tau_{i}^{\top}}\right)-\frac{2(\eta+1)}{\eta-2+\tilde{\varepsilon}_{i t}^{2}}\left(\tilde{\varepsilon}_{i t} \frac{\partial^{2} \tilde{\varepsilon}_{i t}}{\partial \eta \partial \tau_{i}^{\top}}+\frac{\partial \tilde{\varepsilon}_{i t}}{\partial \eta} \frac{\partial \tilde{\varepsilon}_{i t}}{\partial \tau_{i}^{\top}}\right)
\end{aligned}
$$


where

$$
\begin{aligned}
\frac{\partial \tilde{\varepsilon}_{t}^{\top} \tilde{\Theta}_{t}^{-1} \tilde{\varepsilon}_{t}}{\partial \tau_{i}^{\top}} & =2 \tilde{\varepsilon}_{t}^{\top} \tilde{\Theta}_{t}^{-1} \frac{\partial \tilde{\varepsilon}_{t}}{\partial \tau_{i}^{\top}}-2\left(\tilde{\varepsilon}_{t}^{\top} \tilde{\Theta}_{t}^{-1} \otimes \tilde{\varepsilon}_{t}^{\top} \tilde{\Theta}_{t}^{-1}\right) \tilde{M}_{t} \frac{\partial \tilde{\theta}_{t}}{\partial \tau_{i}^{\top}} \\
\frac{\partial \tilde{\Theta}_{t}^{-1} \tilde{\varepsilon}_{t}}{\partial \tau_{i}^{\top}} & =\tilde{\Theta}_{t}^{-1} \frac{\partial \tilde{\varepsilon}_{t}}{\partial \tau_{i}^{\top}}-2\left(\tilde{\varepsilon}_{t}^{\top} \tilde{\Theta}_{t}^{-1} \otimes \tilde{\Theta}_{t}^{-1}\right) \tilde{M}_{t} \frac{\partial \tilde{\theta}_{t}}{\partial \tau_{i}^{\top}}
\end{aligned}
$$

Then

$$
\begin{aligned}
\frac{\partial^{2} l_{t}^{C}}{\partial \eta \partial \delta^{\top}} & =\frac{\partial \tilde{\theta}_{t}^{\top}}{\partial \eta} \frac{\partial \tilde{u}_{t}}{\partial \delta^{\top}}+\tilde{u}_{t}^{\top} \frac{\partial^{2} \tilde{\theta}_{t}}{\partial \eta \partial \delta^{\top}} \\
& +\left\{\frac{\eta+N}{\eta-2+\tilde{\varepsilon}_{t}^{\top} \tilde{\Theta}_{t}^{-1} \tilde{\varepsilon}_{t}}\left(2 \tilde{\varepsilon}_{t}^{\top} \tilde{\Theta}_{t}^{-1} \frac{\partial \tilde{\varepsilon}_{t}}{\partial \eta}-\frac{\tilde{\varepsilon}_{t}^{\top} \tilde{\Theta}_{t}^{-1} \tilde{\varepsilon}_{t}}{\eta-2}\right)-\frac{N+2}{\eta-2}\right\}\left(\eta-2+\tilde{\varepsilon}_{t}^{\top} \tilde{\Theta}_{t}^{-1} \tilde{\varepsilon}_{t}\right)^{-1} \frac{\partial \tilde{\varepsilon}_{t}^{\top} \tilde{\Theta}_{t}^{-1} \tilde{\varepsilon}_{t}}{\partial \delta^{\top}} \\
& +\frac{2(\eta+N)}{\eta-2+\tilde{\varepsilon}_{t}^{\top} \tilde{\Theta}_{t}^{-1} \tilde{\varepsilon}_{t}}\left(\tilde{\varepsilon}_{t}^{\top} \tilde{\Theta}_{t}^{-1} \frac{\partial^{2} \tilde{\varepsilon}_{t}}{\partial \eta \partial \delta^{\top}}+\frac{\partial \tilde{\varepsilon}_{t}^{\top}}{\partial \eta} \frac{\partial \tilde{\Theta}_{t}^{-1} \tilde{\varepsilon}_{t}}{\partial \delta \top}\right)
\end{aligned}
$$

and finally,

$$
\begin{aligned}
\frac{\partial l^{2}}{\partial \eta^{2}} & =\frac{\partial^{2} c(\eta, N)}{\partial \eta^{2}}-N \frac{\partial^{2} c(\eta, 1)}{\partial \eta^{2}}-\frac{1}{2} \sum_{t=1}^{T} \frac{\partial^{2} l_{t}^{C}}{\partial \eta^{2}} \\
\frac{\partial^{2} l_{t}^{C}}{\partial \eta^{2}} & =\frac{\partial \tilde{\theta}_{t}^{\top}}{\partial \eta} \frac{\partial \tilde{u}_{t}}{\partial \eta}+\tilde{u}_{t}^{\top} \frac{\partial^{2} \tilde{\theta}_{t}}{\partial \eta \partial \eta}+\left(\eta-2+\tilde{\varepsilon}_{t}^{\top} \tilde{\Theta}_{t}^{-1} \tilde{\varepsilon}_{t}\right)^{-1} \times \\
& \times\left\{\left(2 \tilde{\varepsilon}_{t}^{\top} \tilde{\Theta}_{t}^{-1} \frac{\partial \tilde{\varepsilon}_{t}}{\partial \eta}-\frac{\tilde{\varepsilon}_{t}^{\top} \tilde{\Theta}_{t}^{-1} \tilde{\varepsilon}_{t}}{\eta-2}\right)\left[1-\frac{N+2}{\eta-2}-\frac{N+\eta}{\eta-2+\tilde{\varepsilon}_{t}^{\top} \tilde{\Theta}_{t}^{-1} \tilde{\varepsilon}_{t}}\left(1+\frac{\partial \tilde{\varepsilon}_{t}^{\top} \tilde{\Theta}_{t}^{-1} \tilde{\varepsilon}_{t}}{\partial \eta}\right)\right]\right. \\
& \left.+2 \frac{N+2}{\eta-2}\left(\tilde{\varepsilon}_{t}^{\top} \tilde{\Theta}_{t}^{-1} \otimes \tilde{\varepsilon}_{t}^{\top} \tilde{\Theta}_{t}^{-1}\right) \tilde{M}_{t} \frac{\partial \tilde{\theta}_{t}}{\partial \eta}+2(\eta+N)\left[\frac{\partial \tilde{\varepsilon}_{t}^{\top} \tilde{\Theta}_{t}^{-1}}{\partial \eta} \frac{\partial \tilde{\varepsilon}_{t}}{\partial \eta}+\tilde{\varepsilon}_{t}^{\top} \tilde{\Theta}_{t}^{-1} \frac{\partial^{2} \tilde{\varepsilon}_{t}}{\partial \eta^{2}}\right]\right\} \\
& -\sum_{i=1}^{N}\left(\eta-2+\tilde{\varepsilon}_{i t}^{2}\right)^{-1} \times \\
\times & \left\{\left(2 \tilde{\varepsilon}_{t} \frac{\partial \tilde{\varepsilon}_{i t}}{\partial \eta}-\frac{\tilde{\varepsilon}_{i t}^{2}}{\eta-2}\right)\left[\frac{\eta-5}{\eta-2}-\frac{1+\eta}{\eta-2+\tilde{\varepsilon}_{i t}^{2}}\left(1+\frac{\partial \tilde{\varepsilon}_{i t}^{2}}{\partial \eta}\right)\right]+2(\eta+1)\left[\left(\frac{\partial \tilde{\varepsilon}_{i t}}{\partial \eta}\right)+\tilde{\varepsilon}_{i t}^{2} \frac{\partial^{2} \tilde{\varepsilon}_{i t}}{\partial \eta^{2}}\right]\right\}
\end{aligned}
$$


where

$$
\begin{aligned}
\frac{\partial \tilde{\varepsilon}_{t}^{\top} \tilde{\Theta}_{t}^{-1} \tilde{\varepsilon}_{t}}{\partial \eta} & =2 \tilde{\varepsilon}_{t}^{\top} \tilde{\Theta}_{t}^{-1} \frac{\partial \tilde{\varepsilon}_{t}}{\partial \eta}-2\left(\tilde{\varepsilon}_{t}^{\top} \tilde{\Theta}_{t}^{-1} \otimes \tilde{\varepsilon}_{t}^{\top} \tilde{\Theta}_{t}^{-1}\right) \tilde{M}_{t} \frac{\partial \tilde{\theta}_{t}}{\partial \eta} \\
\frac{\partial \tilde{\Theta}_{t}^{-1} \tilde{\varepsilon}_{t}}{\partial \eta} & =\tilde{\Theta}_{t}^{-1} \frac{\partial \tilde{\varepsilon}_{t}}{\partial \eta}-2\left(\tilde{\varepsilon}_{t}^{\top} \tilde{\Theta}_{t}^{-1} \otimes \tilde{\Theta}_{t}^{-1}\right) \tilde{M}_{t} \frac{\partial \tilde{\theta}_{t}}{\partial \eta} \\
\frac{\partial \tilde{\theta}_{t}}{\partial \eta} & =\Phi \frac{\partial \tilde{\theta}_{t-1}}{\partial \eta}+K \frac{\partial \tilde{u}_{t-1}}{\partial \eta} \\
\frac{\partial^{2} \tilde{\theta}_{t}}{\partial \eta^{2}} & =\Phi \frac{\partial^{2} \tilde{\theta}_{t-1}}{\partial \eta^{2}}+K \frac{\partial^{2} \tilde{u}_{t-1}}{\partial \eta^{2}}
\end{aligned}
$$

These expressions can be used, as for one-step estimation, to estimate consistently the asymptotic covariance matrix by evaluating it at $\hat{\vartheta}$ and replacing the expectation operators by sample averages. We will use this two-step estimation approach in our empirical application, to which we turn in the following.

\section{Empirical Application}

Modelling the day-to-day changes in the correlation structure of financial assets plays an important role for financial decision making such as portfolio allocation. What we have in mind is an investor who, in a context of near-zero interest rates, considers not only classical assets such as stocks and bonds, but also commodities (oil), safe-haven investments (gold), and alternative investments (fine art or cryptocurrencies). For each market we take a representative index and then model the set of market indices using our multivariate framework. In the following, we first present the data, then the results for the volatility part of the model, and finally the results for the correlation part.

\subsection{Data Description}

We use historical data of daily returns for six representative indices of different investment categories, including both traditional financial assets (i.e. stocks and bonds) as well as commodities and alternative investments (i.e. oil, gold and cryptocurrencies). The sample period spans from August 1, 2014 until December 28, 2018, with the starting 
date determined by the first available data point of the cryptocurrency index CRIX, see Trimborn and Härdle 2018 for details on the construction of CRIX. The symbols and description for the indices are shown in Table 1.

The time series of prices and returns are shown in Figure 2 and Figure 3, respectively. Note the peculiar behavior of the CRIX that showed bubble-like features in 2017 with a

slow erosion thereafter. Table 2 presents the main descriptive statistics for our historical return series of six assets. Alternative investments generally have larger mean returns than stocks or bonds, with higher associated volatilities. The equity index EMU has a much larger skewness and kurtosis than SPX, which is caused by a single abnormal return in 2016. Equity and bond returns are generally negatively skewed, implying a long tail in negative returns, whereas most alternatives are positively skewed indicating large extrema on the positive side. The CRIX is an exception with a negative skewness, which may be explained by the market downturn of 2018. Furthermore, normality is clearly rejected for all assets by classical tests at conventional significance levels.

In Table 3, the sample correlation matrix of historical returns for the six market indices is provided. For financial indices, SPX and EMU are highly correlated with a Pearson correlation coefficient of 0.48 . The negative coefficients between pairs of SPX-SBW and SPX-GOLD agree with the common intuition that bonds and gold often serve as an effective hedge against stock market risk. This characteristic also results in the fact that the correlation between gold and bonds is relatively high. Oil is more closely correlated with the equity index, while correlation with other alternatives is negligible.

\subsection{Volatility estimation results}

We follow the two-step estimation procedure outlined in Section 3, where in the first step, volatility models are fitted to the return series. For each series, we fit a Betat-EGARCH(1,1) model and compare it with a classical $\operatorname{GARCH}(1,1)$. Both models are estimated by maximum likelihood, where we use a Gaussian MLE for GARCH, and a conditional student-t distribution for Beta-t-EGARCH. In order to improve the convergence 
of the optimization algorithm, each return series is standardized by its sample standard deviation. The results are shown in Table 4. All parameter estimates are significantly different from zero using estimated asymptotic standard errors.

Due to the prior standardization of returns, the unconditional volatility of each time series as implied by the estimated model parameters is close to unity. Moreover, there is high persistence in volatility for all time series, confirming the presence of "volatility clustering". Note that the CRIX has the lowest estimated d.o.f. $\eta_{i}=1 / \bar{\eta}_{i}$ which corresponds to its observed high kurtosis and thick tails.

In Figure 4, we plot the estimated volatility series under $\operatorname{GARCH}(1,1)$ against Betat-EGARCH. In general, the estimated volatility series for most assets are close to each other, except for some events such as the outlier in EMU in mid-2016, for which GARCH volatility reacts much stronger than Beta-t-EGARCH. This can be explained by the wellknown saturation of the news impact curve of Beta-t-EGARCH for large shocks as opposed to $\operatorname{GARCH}(1,1)$, see e.g. Figure 1.1 of Harvey 2013.

We now focus on assessing the validity of the assumptions for model Beta-t-EGARCH in terms of residual distributions and score series. Firstly, we test whether the score series is a martingale difference sequence (MDS) using a Box-Pierce test for autocorrelation. The p-values are displayed in Table 5. Since for all six indices the p-values are larger than $5 \%$, we fail to reject the null hypothesis that the score series have zero autocorrelation up to a lag of 30, and the martingale difference property is confirmed. Furthermore, we apply the Box-Pierce test to the standardized residuals and their squares. The results, also reported in Table 5, indicate that significant autocorrelations exist only for the residuals of CRIX at a significance level of $5 \%$, but not at $1 \%$, whereas the squared residuals for all assets are not serially correlated at $5 \%$.

The descriptive statistics of standardized residuals are summarized in Table 6. As expected, the sample means are close to zero and the standard deviations close to one. The only exception is the CRIX whose residuals have a standard deviation of 0.81. Again, this may be caused by some remaining serial correlation in the residuals or outliers. There is remaining excess kurtosis in the standardized residual series, so that a conditional 
leptokurtic distribution provides a better fit to the data than a normal distribution.

Finally, we use the probability integral transformation (PIT) to check how well the assumed conditional distribution fits the data. In Figure 5, we display the histograms of PITs of residual series, assuming student-t and normal distributions respectively. Most of the histograms for the normal distribution have a hump in the middle and pronounced wings in the tails, which corresponds to our finding of leptokurtic standardized residuals in Table 6. Although not perfect, the histograms of the PITs for the student-t distribution are much closer to a uniform distribution than the Gaussian PITs. Introducing skewness may be beneficial, but we have not further pursued this in our analysis and leave it as a potential extension in the future.

In the next section, we move on to the second step of the two-step estimation and focus on the correlation part of the model, taking the standardized residual series of the univariate volatility models as input for the correlation model.

\subsection{Results for the conditional correlations}

We now turn to the results for the second step estimation regarding the conditional correlation matrix. First, we want to test the reliability of our assumption of diagonal $K$ and $\Phi$ matrices. As it is infeasible to estimate a model with six asset indices and full

matrices $K$ and $\Phi$ (it would have 466 parameters), we chose five subsets composed of three out of six assets with relatively high correlations, and perform likelihood-ratio tests to assess the goodness of fit for the diagonal case $\left(H_{0}\right)$ with respect to the general one. We concluded that for all pairs at significance level of $\alpha=5 \%, H_{0}$ can not be rejected, which corroborates our assumption of diagonal parameter matrices used for the six asset indices.

The estimated parameters for the models with diagonal $K$ and $\Phi$ are listed in Table 7 . All estimated $\kappa_{i}$ coefficients are positive and close to zero, while the estimated $\phi_{i}$ are close to one. Similar to the volatility case, the correlation series are characterized by stationarity but high persistence. Even with the diagonality constraints we still have 46 
parameters to estimate. To simplify the model, we additionally consider five further model restrictions. Note first from the estimates in Table 7 that they are quite similar across $\theta_{i t}$ for different $i$, which motivates setting them equal. Table 8 provides a summary for the restricted models and corresponding number of parameters. To economize on space, we do not provide the estimates of the restricted models and only provide summarized performance measures in the following.

Figure 6 plots the estimated correlation series for selected five pairs of asset indices with absolute value of sample correlation coefficient being higher than $15 \%$ for the first two models. The five pairs are SPX-SBW; SPX-EMU; SPX-OIL; SBW-GOLD and EMU-OIL. While the overall level of estimated correlations is about the same for both models, there is a difference in the estimated dynamics: Model 2 restricts all dynamics to be the same, while Model 1 allows for different dynamics. Effectively, this leads to more erratic correlation series for the pairs SPX-SBW and SPX-EMU using Model 1 than using Model 2, while the converse holds for SPX-OIL. For SBW-GOLD, estimated correlations using Model 1 are rather stable over time while those of Model 2 appear to have a positive trend in the first half of the sample. Finally, the estimated correlations of EMU-OIL are rather similar for both models.

In terms of model diagnostics, we first apply portmanteau tests to the correlation score series. The results are reported in Table 9. For all six assets, there is no evidence against the martingale difference property at conventional significance levels. Furthermore, we perform likelihood-ratio tests of the restricted models with respect to the diagonal one. The results are displayed in Table 9. It can be observed that at a significance level of $1 \%$, we cannot reject $H_{0}$ for model 2 and model 3 , while for the rest $H_{0}$ is rejected. We additionally report the Akaike and Bayesian information criteria (AIC and BIC), to quantify the model performance in terms of goodness-of-fit and model complexity. Based on the summarized statistics in Table 9, it can be concluded that the most parsimonious model 6 with only 3 parameters has the minimum BIC, while according to AIC, Model 2 with 18 parameters should be selected. In both cases, this is evidence in favor of restricting the diagonal elements of $K$ and those of $\Phi$ to be equal, which reduces the 
parameter dimension substantially.

To summarize, this also suggests that the dynamics of the individual elements of $\theta_{i}$ can be assumed to be the same across components, and that the only difference occurs in the intercept terms $\omega_{i}$. In higher dimensions, we conjecture that similar parameter restrictions are reasonable and allow us to obtain computationally feasible results in cases of several hundreds of assets. This actually corresponds to a similar observation made by Caporin and McAleer 2012 for the classes of BEKK and DCC models, for which the "scalar" versions of the models are preferred in high dimensions to avoid the curse of dimensionality.

\section{Conclusions}

The proposed score-driven correlation model ensures positivity of the conditional correlation matrix while offering high flexibility in the modelling of the conditional distribution and dynamics. In the empirical application we have shown that the model fits well a set of financial and alternative assets, passing various diagnostic tests for the distribution and dynamics. We thus expect it to perform well compared to alternative multivariate volatility models in terms of out-of-sample forecasting of correlations and financial applications such as portfolio selection, and leave this for further empirical analyses.

Many generalizations are possible, for example using generalized student-t distributions as in Harvey and Lange (2017), and distributions allowing for skewness as in Bauwens and Laurent (2005) or Harvey and Sucarrat (2014). Rather than models based on the student-t distribution, one may also generalize the Gamma-GED-EGARCH to the multivariate case. Future work may also address specification tests in high dimensions, for example clustering of parameters characterizing the dynamics of the log correlation matrix. We have discussed two extreme cases in the empirical example, the unrestricted case and the one setting all parameters equal, but developing strategies to specify intermediate cases would seem reasonable as well. 


\section{Acknowledgements}

We are grateful for discussions with participants at the INET Cambridge workshop on score driven time series models in March 2019. Both authors acknowledge financial support of the ARC research contract 18/23-089 of the Belgian government. 


\section{Appendices}

\section{A Proofs}

\section{A.1 Proof of Theorem 1}

Proof. First, we have

$$
\begin{aligned}
d L(\delta) & =-\frac{1}{2} \sum_{t=1}^{T}\left\{d \log \left|\Theta_{t}\right|-\frac{\eta+N}{\eta-2+\varepsilon_{t}^{\top} \Theta_{t}^{-1} \varepsilon_{t}} \operatorname{Tr}\left(\varepsilon_{t} \varepsilon_{t}^{\top} d \Theta_{t}^{-1}\right)\right\} \\
& =-\frac{1}{2} \sum_{t=1}^{T} \operatorname{Tr}\left\{\Theta_{t}^{-1} d \Theta_{t}-\frac{\eta+N}{\eta-2+\varepsilon_{t}^{\top} \Theta_{t}^{-1} \varepsilon_{t}} \varepsilon_{t} \varepsilon_{t}^{\top} \Theta_{t}^{-1} d \Theta_{t} \Theta_{t}^{-1}\right\} \\
& =\frac{1}{2} \sum_{t=1}^{T} \operatorname{Tr}\left\{\left(\frac{\eta+N}{\eta-2+\varepsilon_{t}^{\top} \Theta_{t}^{-1} \varepsilon_{t}} \Theta_{t}^{-1} \varepsilon_{t} \varepsilon_{t}^{\top} \Theta_{t}^{-1}-\Theta_{t}^{-1}\right) d \Theta_{t}\right\} \\
& =\frac{1}{2} \sum_{t=1}^{T} \operatorname{vec}\left(\frac{\eta+N}{\eta-2+\varepsilon_{t}^{\top} \Theta_{t}^{-1} \varepsilon_{t}} \Theta_{t}^{-1} \varepsilon_{t} \varepsilon_{t}^{\top} \Theta_{t}^{-1}-\Theta_{t}^{-1}\right)^{\top} d \operatorname{vec} \Theta_{t}
\end{aligned}
$$

Now, using a result of Linton and McCrorie (1995) for the differential of the matrix exponential, we can write

$$
d \mathrm{vec} \Theta_{t}=A_{t} d \mathrm{vec} \log \Theta_{t},
$$

and with the definition of the permutation matrix $P$,

$$
d \operatorname{vec} \Theta_{t}=A_{t} P\left(\begin{array}{l}
d \operatorname{diag}\left(\log \Theta_{t}\right) \\
d \operatorname{vecl}\left(\log \Theta_{t}\right)
\end{array}\right)
$$

Now, vecl $\left(\log \Theta_{t}\right)=\theta_{t}$, and by Archakova and Hansen (2018), $\operatorname{diag}\left(\log \Theta_{t}\right)$ is a unique function of $\theta_{t}$, for which no analytical form exists for $N>4$. However, using the proof of Proposition 3 of Archakova and Hansen (2018), we have an analytical expression for the differential of this function, given by

$$
d \operatorname{diag}\left(\log \Theta_{t}\right)=-\left(E_{d} A_{t} E_{d}^{\top}\right)^{-1} E_{d} A_{t} E_{s}^{\top} d \theta_{t}
$$

which completes the stated result. 
The expressions given in Remark 1 for the Gaussian distribution, i.e. for the limiting case $\eta \rightarrow \infty$, can be obtaine easily. First, using Stirling's approximation, note that

$$
\begin{aligned}
\lim _{\eta \rightarrow \infty} & {\left[T \log \left(\Gamma\left(\frac{\eta+N}{2}\right)\right)-T \log \left(\Gamma\left(\frac{\eta}{2}\right)\right)-\frac{T N}{2} \log ((\eta-2) \pi)\right] } \\
= & \lim _{\eta \rightarrow \infty} T \log \frac{\Gamma\left(\frac{\eta+N}{2}\right)}{\Gamma\left(\frac{\eta}{2}\right)((\eta-2) \pi)^{N / 2}} \\
= & T \log (2 \pi)^{-N / 2}=-\frac{T N}{2} \log (2 \pi)
\end{aligned}
$$

And for the score function, it can be easily shown that

$$
\begin{aligned}
p \lim _{\eta \rightarrow \infty} \quad & -\frac{1}{2} \sum_{t=1}^{T}\left\{\log \left|\Theta_{t}\right|+(\eta+N) \log \left(1+\frac{1}{\eta-2} \varepsilon_{t}^{\top} \Theta_{t}^{-1} \varepsilon_{t}\right)\right\} \\
= & p \lim _{\eta \rightarrow \infty}-\frac{1}{2} \sum_{t=1}^{T}\left\{\log \left|\Theta_{t}\right|+\frac{\eta+N}{\eta-2} \varepsilon_{t}^{\top} \Theta_{t}^{-1} \varepsilon_{t}\right\} \\
= & -\frac{1}{2} \sum_{t=1}^{T}\left\{\log \left|\Theta_{t}\right|+\varepsilon_{t}^{\top} \Theta_{t}^{-1} \varepsilon_{t}\right\}
\end{aligned}
$$

Thus, we arrive at the expression of the log likelihood function for the multivariate normal distribution. Furthermore, the score can be obtained directly by taking the probability limit of $\frac{\eta+N}{\eta-2+\varepsilon_{t}^{\top} \Theta_{t}^{-1} \varepsilon_{t}}$ as $\eta \rightarrow \infty$, which is equal to 1 .

\section{A.2 Proof of Theorem 2}

We first need to show that, under our conditions, $\theta_{t}$ and its derivatives up to order 3 , evaluated at the true parameters, are stationary and ergodic processes. The process $\theta_{t}$ satisfies the stochastic recurrence equation (SRE) in (12) which by Theorem 2.8 of Straumann and Mikosch (2006) is stationary and ergodic under the contraction condition of Assumption (A6). The compactness of $\Omega$ then implies consistency by Lemma 1 of Jensen and Rahbek (2004).

The process $\partial \theta_{t} / \partial \delta$ permits an SRE as in (16). It is stationary and ergodic if $\mathrm{E}\left[\log \left|x_{t}\right|\right]<$ 0 , see Straumann and Mikosch (2006). This is implied by Assumption (A5), i.e. $\mathrm{E}\left[x_{t}^{2}\right]<1$, using Jensen's inequality. 
The process $\frac{\partial^{2} \theta_{t}}{\partial \delta \partial \delta \mathrm{T}}$ also permits an SRE:

$$
\frac{\partial^{2} \theta_{t}}{\partial \delta \partial \delta \top}=C_{t-1}+x_{t-1} \frac{\partial^{2} \theta_{t-1}}{\partial \delta \partial \delta^{\top}}
$$

where $C_{t}=\frac{\partial z_{t}}{\partial \delta^{\top}}+\frac{\partial \theta_{t}}{\partial \delta} \frac{\partial x_{t}}{\partial \delta^{\top}}$. Again, stationary and ergodicity are implied by Assumption (A5). The same argument applies to the third derivatives of $\theta_{t}$.

We then obtain asymptotic normality for the score evaluated at the true parameter, invoking the central limit theorem for martingale difference sequences of Brown (1971), i.e.

$$
T^{-1 / 2} \sum_{t=1}^{T} \frac{\partial l_{t}\left(\vartheta_{0}\right)}{\partial \vartheta} \rightarrow_{d} N\left(0, I\left(\vartheta_{0}\right)\right) .
$$

This requires that $\mathrm{E}\left[\left(\partial \theta_{i t} / \partial \vartheta_{j}\right)^{2}\right]<\infty$ for $i=1, \ldots, N^{*}$ and $j=1 \ldots, \operatorname{dim}(\vartheta)$, which is implied by Assumption (A5), see Theorem 1 of Harvey (2013).

Moreover, we have

$$
-\frac{1}{T} \sum_{t=1}^{T} \frac{\partial^{2} l_{t}\left(\vartheta_{0}\right)}{\partial \vartheta \partial \vartheta^{\top}} \rightarrow_{p} I\left(\vartheta_{0}\right)
$$

as in Proposition 5 of Harvey (2013). It suffices to check that, for a neighborhood $N\left(\vartheta_{0}\right)$ of the true parameter,

$$
\mathrm{E} \sup _{\vartheta \in N\left(\vartheta_{0}\right)}\left\|\frac{\partial \operatorname{vec}}{\partial \vartheta^{\prime}} \frac{\partial^{2} l_{t}(\vartheta)}{\partial \vartheta \partial \vartheta^{\prime}}\right\|<\infty .
$$

For the volatility part this has been shown before, see e.g. Theorem 3 of Harvey (2013), so we concentrate on the correlation part. We have

$$
\begin{aligned}
\frac{\partial \operatorname{vec}}{\partial \delta^{\top}} \frac{\partial^{2} l_{t}(\delta)}{\partial \delta \partial \delta^{\top}} & =\left(I_{m} \otimes u_{t}^{\top} \otimes I_{m}\right) \frac{\partial \operatorname{vec}}{\partial \delta^{\top}} \frac{\partial \operatorname{vec}}{\partial \delta^{\top}} \frac{\partial \theta_{t}^{\top}}{\partial \delta}+\left(\frac{\partial \operatorname{vec}^{\top}}{\partial \delta} \frac{\partial \theta_{t}^{\top}}{\partial \delta} \otimes I_{m}\right)\left(I_{N^{*}} \otimes \operatorname{vec}\left(I_{m}\right)\right) \frac{\partial u_{t}}{\partial \delta^{\top}} \\
& +\left(\frac{\partial u_{t}^{\top}}{\partial \delta} \otimes I_{m}\right) \frac{\partial \operatorname{vec}}{\partial \delta^{\top}} \frac{\partial \theta_{t}^{\top}}{\partial \delta}+\left(I_{m} \otimes \frac{\partial \theta_{t}^{\top}}{\partial \delta}\right) \frac{\partial \operatorname{vec}}{\partial \delta^{\top}} \frac{\partial u_{t}}{\partial \delta^{\top}}
\end{aligned}
$$

with $m=\operatorname{dim}(\delta)$. The first term on the right hand side of (19) depends on the third order derivatives of the correlation parameter with respect to $\delta$. A typical element of this matrix is given by

$$
\begin{aligned}
\frac{\partial^{3} \theta_{t}(\delta)}{\partial \delta_{i} \partial \delta_{j} \partial \delta_{k}} & =\frac{\partial^{2} z_{i, t-1}}{\partial \theta_{j} \partial \theta_{k}}+\frac{\partial x_{t-1}}{\partial \delta_{j}} \frac{\partial^{2} \theta_{t-1}}{\partial \delta_{i} \partial \delta_{k}}+\frac{\partial^{2} x_{t-1}}{\partial \delta_{j} \partial \delta_{k}} \frac{\partial \theta_{t-1}}{\partial \delta_{i}}+\frac{\partial x_{t-1}}{\partial \delta_{k}} \frac{\partial^{2} \theta_{t-1}}{\partial \delta_{i} \partial \delta_{j}}+x_{t-1} \frac{\partial^{3} \tilde{\theta}_{t-1}(\delta)}{\partial \delta_{i} \partial \delta_{j} \partial \delta_{k}} \\
& =\sum_{j=1}^{\infty} \zeta_{t-j} \prod_{i=1}^{j-1} x_{t-i}
\end{aligned}
$$


where $z_{i t}$ denotes the $i$-th column of $z_{t}$, and $\zeta_{t}=\frac{\partial^{2} z_{i, t-1}}{\partial \theta_{j} \partial \theta_{k}}+\frac{\partial x_{t-1}}{\partial \delta_{j}} \frac{\partial^{2} \theta_{t-1}}{\partial \delta_{i} \partial \delta_{k}}+\frac{\partial^{2} x_{t-1}}{\partial \delta_{j} \partial \delta_{k}} \frac{\partial \theta_{t-1}}{\partial \delta_{i}}+$ $\frac{\partial x_{t-1}}{\partial \delta_{k}} \frac{\partial^{2} \theta_{t-1}}{\partial \delta_{i} \partial \delta_{j}}$. Each term of $\zeta_{t}$ can be uniformly bounded in a neighborhood of $\delta_{0}$ and a repeated application of the $c_{r}$ inequality yields $\operatorname{Esup}_{\delta \in N\left(\delta_{0}\right)}\left|\zeta_{t}\right|<\infty$. Note that $\left|x_{t}\right|$ is a stationary ergodic sequence of nonnegative random variables with E $\log \left|x_{t}\right|<0$, which is implied by Assumption (A5). Thus, by Lemma 2.4 of Straumann and Mikosch (2006), $\prod_{i=1}^{t-1}\left|x_{t-i}\right| \rightarrow_{e . a . s .} 0$, meaning that there exists a $\gamma>1$ s.t. $\gamma^{t} \prod_{i=1}^{t-1}\left|x_{t-i}\right| \rightarrow_{a . s .}$ 0. This implies that $\mathrm{E} \prod_{i=1}^{t-1}\left|x_{t-i}\left(\bar{\delta}_{t-i}\right)\right|=O\left(\rho^{t}\right)$, which in turn implies that the infinite sum in (20) is convergent and we obtain

$$
\mathrm{E} \sup _{\theta \in N\left(\theta_{0}\right)}\left|\frac{\partial^{3} \theta_{t}(\delta)}{\partial \delta_{i} \partial \delta_{j} \partial \delta_{k}}\right|<\infty .
$$

The remaining terms on the right hand side of (19) can be bounded in a similar way. Together with corresponding results for the volatility part, this implies (18).

Finally, tedious but straightforward calculations yield the expression of the information matrix using results of Fiorentini et al. (2003). The details are omitted here but available upon request.

\section{A.3 Proof of Theorem 3}

The proof of consistency and asymptotic normality is essentially the same as that of Theorem 2, noting that stationarity and ergodicity of the process $\tilde{\theta}_{t}$ hold under the same conditions as for the process $\theta_{t}$. The form of the asymptotic covariance matrix of the inference function for margins approach is based on Joe (2005), and the components of $\mathcal{I}$ follow by straightforward calculations, which are again omitted to economize on space, but available upon request. 


\section{B Example: The bivariate Gaussian case}

This section shows that our model reduces to that of Harvey (2013, Section 7.3.1) in the bivariate Gaussian case. It also serves to illustrate the structure of the involved matrix expressions. Let $N=2$. For $\Theta$ and $\log \Theta$, see Example 1 above. We have $A_{t}=\left(\Gamma_{t} \otimes \Gamma_{t}\right) \Xi_{t}\left(\Gamma_{t} \otimes \Gamma_{t}\right)^{\top}$ with $\operatorname{diag}\left(\Xi_{t}\right)=\left(1+\rho, 2 \rho / \log \frac{1+\rho}{1-\rho}, 2 \rho / \log \frac{1+\rho}{1-\rho}, 1-\rho\right)$ and

$$
\Gamma_{t} \otimes \Gamma_{t}=\frac{1}{2}\left(\begin{array}{cccc}
1 & 1 & 1 & 1 \\
1 & -1 & 1 & -1 \\
1 & 1 & -1 & -1 \\
1 & -1 & -1 & 1
\end{array}\right)
$$

which gives

$$
A_{t}=\frac{1}{2}\left(\begin{array}{cccc}
1+\frac{2 \rho}{\log \frac{1+\rho}{1-\rho}} & \rho & \rho & 1-\frac{2 \rho}{\log \frac{1+\rho}{1-\rho}} \\
\rho & 1+\frac{2 \rho}{\log \frac{1+\rho}{1-\rho}} & 1-\frac{2 \rho}{\log \frac{1+\rho}{1-\rho}} & \rho \\
\rho & 1-\frac{2 \rho}{\log \frac{1+\rho}{1-\rho}} & 1+\frac{2 \rho}{\log \frac{1+\rho}{1-\rho}} & \rho \\
1-\frac{2 \rho}{\log \frac{1+\rho}{1-\rho}} & \rho & \rho & 1+\frac{2 \rho}{\log \frac{1+\rho}{1-\rho}}
\end{array}\right)
$$

The elimination matrices are $E_{l}=(0,1,0,0), E_{u}=(0,0,1,0)$, and

$$
E_{d}=\left(\begin{array}{llll}
1 & 0 & 0 & 0 \\
0 & 0 & 0 & 1
\end{array}\right)
$$

such that

$$
\begin{gathered}
E_{d} A_{t} E_{d}^{\top}=\frac{1}{2}\left(\begin{array}{cc}
1+\frac{2 \rho}{\log \frac{1+\rho}{1-\rho}} & 1-\frac{2 \rho}{\log \frac{1+\rho}{1-\rho}} \\
1-\frac{2 \rho}{\log \frac{1+\rho}{1-\rho}} & 1+\frac{2 \rho}{\log \frac{1+\rho}{1-\rho}}
\end{array}\right), \quad E_{d} A_{t} E_{s}^{\top}=\left(\begin{array}{l}
\rho \\
\rho
\end{array}\right) \\
\left(E_{d} A_{t} E_{d}^{\top}\right)^{-1}=\frac{\log \frac{1+\rho}{1-\rho}}{4 \rho}\left(\begin{array}{cc}
1+\frac{2 \rho}{\log \frac{1+\rho}{1-\rho}} & \frac{2 \rho}{\log \frac{1+\rho}{1-\rho}}-1 \\
\frac{2 \rho}{\log \frac{1+\rho}{1-\rho}}-1 & 1+\frac{2 \rho}{\log \frac{1+\rho}{1-\rho}}
\end{array}\right) \\
-\left(E_{d} A_{t} E_{d}^{\top}\right)^{-1} E_{d} A_{t} E_{s}^{\top}=-\frac{\log \frac{1+\rho}{1-\rho}}{4 \rho}\left(\begin{array}{cc}
1+\frac{2 \rho}{\log \frac{1+\rho}{1-\rho}} & \frac{2 \rho}{\log \frac{1+\rho}{1-\rho}}-1 \\
\frac{2 \rho}{\log \frac{1+\rho}{1-\rho}-1} & 1+\frac{2 \rho}{\log \frac{1+\rho}{1-\rho}}
\end{array}\right)\left(\begin{array}{l}
\rho \\
\rho
\end{array}\right)=\left(\begin{array}{c}
-\rho \\
-\rho
\end{array}\right)
\end{gathered}
$$


Note that this can be obtained directly from Example 1, where the diagonal elements of $\log \Theta$ are $\frac{1}{2} \log \left(1-\rho^{2}\right)$, and the off-diagonal element is $\gamma:=\frac{1}{2} \log \frac{1+\rho}{1-\rho}$, or $\rho=\frac{\exp (2 \gamma)-1}{\exp (2 \gamma)+1}$. Hence,

$$
\frac{\partial}{\partial \rho} \frac{1}{2} \log \left(1-\rho^{2}\right) \frac{\partial \rho}{\partial \gamma}=\frac{-\rho}{1-\rho^{2}} \frac{4 \exp (2 \gamma)}{(\exp (2 \gamma)+1)^{2}}=\frac{-2 \rho}{1-\rho^{2}}\left(1-\rho^{2}\right)=-2 \rho .
$$

Together with the factor $1 / 2$ in $u_{t}$, we obtain the same result.

The permutation matrix $P$ is given by

$$
P=\left(\begin{array}{lll}
1 & 0 & 0 \\
0 & 0 & 1 \\
0 & 0 & 1 \\
0 & 1 & 0
\end{array}\right)
$$

Then,

$$
\left(\begin{array}{c}
-\left(E_{d} A_{t} E_{d}^{\top}\right)^{-1} E_{d} A_{t} E_{s}^{\top} \\
I_{N(N-1) / 2}
\end{array}\right)^{\top} P^{\top}=\left(\begin{array}{lll}
-\rho & -\rho & 1
\end{array}\right)\left(\begin{array}{llll}
1 & 0 & 0 & 0 \\
0 & 0 & 0 & 1 \\
0 & 1 & 1 & 0
\end{array}\right)=\left(\begin{array}{llll}
-\rho & 1 & 1 & -\rho
\end{array}\right)
$$

and

$$
\begin{aligned}
\left(\begin{array}{llll}
-\rho & 1 & 1 & -\rho
\end{array}\right) A_{t} & =\frac{1}{2}\left(\begin{array}{llll}
-\rho & 1 & 1 & -\rho
\end{array}\right)\left(\begin{array}{cccc}
1+\frac{2 \rho}{\log \frac{1+\rho}{1-\rho}} & \rho & \rho & 1-\frac{2 \rho}{\log \frac{1+\rho}{1-\rho}} \\
\rho & 1+\frac{2 \rho}{\log \frac{1+\rho}{1-\rho}} & 1-\frac{2 \rho}{\log \frac{1+\rho}{1-\rho}} & \rho \\
\rho & 1-\frac{2 \rho}{\log \frac{1+\rho}{1-\rho}} & 1+\frac{2 \rho}{\log \frac{1+\rho}{1-\rho}} & \rho \\
1-\frac{2 \rho}{\log \frac{1+\rho}{1-\rho}} & \rho & \rho & 1+\frac{2 \rho}{\log \frac{1+\rho}{1-\rho}}
\end{array}\right) \\
& =\left(\begin{array}{llll}
0 & 1-\rho^{2} & 1-\rho^{2} & 0
\end{array}\right)
\end{aligned}
$$

Finally,

$$
\operatorname{vec}\left(\Theta^{-1} \varepsilon_{t} \varepsilon_{t}^{\top} \Theta^{-1}\right)=\frac{1}{\left(1-\rho^{2}\right)^{2}}\left(\begin{array}{c}
\varepsilon_{1 t}^{2}-2 \rho \varepsilon_{1 t} \varepsilon_{2 t}+\rho^{2} \varepsilon_{2 t}^{2} \\
\left(1+\rho^{2}\right) \varepsilon_{1 t} \varepsilon_{2 t}-\rho\left(\varepsilon_{1 t}^{2}+\varepsilon_{2 t}^{2}\right) \\
\left(1+\rho^{2}\right) \varepsilon_{1 t} \varepsilon_{2 t}-\rho\left(\varepsilon_{1 t}^{2}+\varepsilon_{2 t}^{2}\right) \\
\rho^{2} \varepsilon_{1 t}^{2}-2 \rho \varepsilon_{1 t} \varepsilon_{2 t}+\varepsilon_{2 t}^{2}
\end{array}\right), \quad \operatorname{vec}\left(\Theta^{-1}\right)=\frac{1}{1-\rho^{2}}\left(\begin{array}{c}
1 \\
-\rho \\
-\rho \\
1
\end{array}\right)
$$


such that

$$
\left(\begin{array}{llll}
0 & 1-\rho^{2} & 1-\rho^{2} & 0
\end{array}\right) \operatorname{vec}\left(\Theta^{-1} \varepsilon_{t} \varepsilon_{t}^{\top} \Theta^{-1}-\Theta^{-1}\right)=\frac{2}{1-\rho^{2}}\left\{\left(1+\rho^{2}\right) \varepsilon_{1 t} \varepsilon_{2 t}-\rho\left(\varepsilon_{1 t}^{2}+\varepsilon_{2 t}^{2}\right)\right\}+2 \rho
$$

and

$$
u_{t}=\frac{1}{1-\rho^{2}}\left\{\left(1+\rho^{2}\right) \varepsilon_{1 t} \varepsilon_{2 t}-\rho\left(\varepsilon_{1 t}^{2}+\varepsilon_{2 t}^{2}\right)\right\}+\rho
$$

which is the score given by Harvey (2013, Section 7.3.1) for the bivariate Gaussian case.

He uses the parametrization $\gamma=\frac{1}{2} \frac{1+\rho}{1-\rho}$ and

$$
g=\frac{\exp \gamma+\exp (-\gamma)}{2}, \quad \bar{g}=\frac{\exp \gamma-\exp (-\gamma)}{2}
$$

so that

$$
u_{t}=\left(2 g^{2}-1\right) \varepsilon_{1 t} \varepsilon_{2 t}-g \bar{g}\left(\varepsilon_{1 t}^{2}+\varepsilon_{2 t}^{2}\right)+\frac{\bar{g}}{g} .
$$

For the information matrix of Theorem 2, the expressions reduce to

$$
\begin{aligned}
I_{\delta \delta} & =\frac{1}{2} \mathbb{E}\left[\frac{\partial \theta_{t}^{\top}}{\partial \delta} M_{t}\left(\Theta_{t}^{-1} \otimes \Theta_{t}^{-1}\right) M_{t}^{\top} \frac{\partial \theta_{t}}{\partial \delta^{\top}}\right] \\
I_{\tau \tau} & =\frac{1}{2} \mathbb{E}\left[\frac{\partial \operatorname{vec}\left(H_{t}\right)^{\top}}{\partial \tau}\left(H_{t}^{-1} \otimes H_{t}^{-1}\right) \frac{\partial \operatorname{vec}\left(H_{t}\right)}{\partial \tau^{\top}}\right] \\
I_{\tau \delta} & =\frac{1}{2} \mathbb{E}\left[\frac{\partial \operatorname{vec}\left(H_{t}\right)^{\top}}{\partial \tau}\left(H_{t}^{-1} \otimes H_{t}^{-1}\right)\left(D_{t} \otimes D_{t}\right) M_{t}^{\top} \frac{\partial \theta_{t}}{\partial \delta^{\top}}\right]
\end{aligned}
$$

With $M_{t}=\left(1-\rho^{2}\right)\left(\begin{array}{llll}0 & 1 & 1 & 0\end{array}\right)$, straightforward calculations show that $\frac{1}{2} M_{t}\left(\Theta_{t}^{-1} \otimes \Theta_{t}^{-1}\right) M_{t}^{\top}=$ $\left(1+\rho^{2}\right)=2-1 / g^{2}$, and

$$
I_{\delta \delta}=\mathbb{E}\left[\left(2-1 / g^{2}\right) \frac{\partial \theta_{t}^{\top}}{\partial \delta} \frac{\partial \theta_{t}}{\partial \delta^{\top}}\right]
$$

Furthermore,

$H_{t}=\left(\begin{array}{cc}\exp \left(2 h_{1}\right) & \rho \exp \left(h_{1}+h_{2}\right) \\ \rho \exp \left(h_{1}+h_{2}\right) & \exp \left(2 h_{2}\right)\end{array}\right), \quad H_{t}^{-1}=\frac{1}{\left(1-\rho^{2}\right)}\left(\begin{array}{cc}\exp \left(-2 h_{1}\right) & -\rho \exp \left(-h_{1}-h_{2}\right) \\ -\rho \exp \left(-h_{1}-h_{2}\right) & \exp \left(-2 h_{2}\right)\end{array}\right)$

and

$$
\frac{\partial \operatorname{vec}\left(H_{t}\right)}{\partial \tau^{\top}}=\frac{\partial \operatorname{vec}\left(H_{t}\right)}{\partial \varpi^{\top}} \frac{\partial \varpi}{\partial \tau^{\top}}, \quad \frac{\partial \operatorname{vech}\left(H_{t}\right)}{\partial \varpi^{\top}}=\left(\begin{array}{ccc}
2 \exp \left(2 h_{1}\right) & 0 & 0 \\
\rho \exp \left(h_{1}+h_{2}\right) & \rho \exp \left(h_{1}+h_{2}\right) & \exp \left(h_{1}+h_{2}\right) \\
0 & 2 \exp \left(2 h_{2}\right) & 0
\end{array}\right)
$$


where $\varpi:=\left(h_{1}, h_{2}, \rho\right)^{\top}$. Straightforward calculations show that

$$
\frac{1}{2} \frac{\partial \operatorname{vec}\left(H_{t}\right)^{\top}}{\partial \varpi}\left(H_{t}^{-1} \otimes H_{t}^{-1}\right) \frac{\partial \operatorname{vec}\left(H_{t}\right)}{\partial \varpi^{\top}}=\left(\begin{array}{ccc}
1+g^{2} & 1-g^{2} & -\bar{g} / g \\
1-g^{2} & 1+g^{2} & -\bar{g} / g \\
-\bar{g} / g & -\bar{g} / g & 2-1 / g^{2}
\end{array}\right)=: J
$$

which is the matrix given in Proposition 40 of Harvey (2013). Thus,

$$
I_{\tau \tau}=\mathbb{E}\left[\frac{\partial \varpi^{\top}}{\partial \tau} J \frac{\partial \varpi}{\partial \tau^{\top}}\right]
$$

Similarly, we obtain

$$
I_{\tau \delta}=\mathbb{E}\left[\frac{\partial \varpi^{\top}}{\partial \tau} J_{3} \frac{\partial \theta}{\partial \delta^{\top}}\right]
$$

where $J_{3}$ is the third column of $J$. 


\section{Tables and Figures}

Table 1: Information and description for six assets

\begin{tabular}{lll}
\hline \hline & SECURITY & DESCRIPTION \\
\hline SPX & SPX Index & S\&P 500 Index \\
SBW & SBWGU Index & FTSE World Government Bond Index \\
& & (WGBI) USD \\
EMU & NDDUEMU Index & MSCI EMU Net Total Return Index \\
OIL & CL1 Comdty & Generic 1st Crude Oil; WTI \\
GOLD & GOLDLNPM Index & LBMA Gold Price PM USD \\
CRIX & CRIX & Cryptocurrency Index \\
\hline \hline
\end{tabular}

Table 2: Descriptive statistics for historical returns

\begin{tabular}{ccccccc}
\hline \hline & SPX & SBW & EMU & OIL & GOLD & CRIX \\
\hline N.Obs & 835 & 835 & 835 & 835 & 835 & 835 \\
Minimum & -0.0383 & -0.0194 & -0.0998 & -0.0907 & -0.0319 & -0.2384 \\
Maximum & 0.0383 & 0.0181 & 0.0397 & 0.1162 & 0.0420 & 0.1985 \\
Mean & 0.0003 & -0.0000 & 0.0002 & 0.0006 & -0.0000 & 0.0007 \\
Stdev & 0.0083 & 0.0038 & 0.0106 & 0.0236 & 0.0083 & 0.0426 \\
Skewness & -0.5023 & -0.0728 & -0.9404 & 0.2468 & 0.2769 & -0.6426 \\
Kurtosis & 2.7477 & 1.8548 & 9.7496 & 2.1656 & 2.5535 & 5.5011 \\
Sharpe Ratio & 0.0384 & -0.0031 & 0.0231 & 0.0237 & -0.0014 & 0.0160 \\
Beta & 0.9965 & -0.0370 & 0.7604 & 1.7393 & -0.0194 & 2.1070 \\
\hline \hline
\end{tabular}

The kurtosis in the table is excess kurtosis. 
Table 3: The sample correlation matrix of returns

\begin{tabular}{ccccccc}
\hline & SPX & SBW & EMU & OIL & GOLD & CRIX \\
\hline SPX & 1.0000 & -0.1727 & 0.4830 & 0.2826 & -0.1000 & -0.0032 \\
SBW & -0.1727 & 1.0000 & 0.0375 & -0.0431 & 0.6148 & 0.0213 \\
EMU & 0.4830 & 0.0375 & 1.0000 & 0.2628 & -0.0477 & 0.0205 \\
OIL & 0.2826 & -0.0431 & 0.2628 & 1.0000 & 0.0098 & -0.0160 \\
GOLD & -0.1000 & 0.6148 & -0.0477 & 0.0098 & 1.0000 & 0.0521 \\
CRIX & -0.0032 & 0.0213 & 0.0205 & -0.0160 & 0.0521 & 1.0000 \\
\hline \hline
\end{tabular}

Table 4: Parameter estimates of the volatility models

\begin{tabular}{cccccccc}
\hline \hline & \multicolumn{3}{c}{ GARCH(1,1) } & \multicolumn{4}{c}{ Beta-t-EGARCH(1,1) } \\
\hline \multirow{2}{*}{ SPX } & $\omega_{i}$ & $\alpha_{i}$ & $\beta_{i}$ & $\omega_{i}$ & $\kappa_{i}$ & $\phi_{i}$ & $\bar{\eta}_{i}$ \\
& 0.08262 & 0.19490 & 0.72475 & -0.00502 & 0.11656 & 0.93421 & 0.19080 \\
& $(0.00054)$ & $(0.00107)$ & $(0.00131)$ & $(0.00025)$ & $(0.00068)$ & $(0.00082)$ & $(0.00125)$ \\
\hline \multirow{2}{*}{ SBW } & 0.00166 & 0.03019 & 0.96762 & -0.00070 & 0.01912 & 0.99480 & 0.11411 \\
& $(0.00010)$ & $(0.00028)$ & $(0.00033)$ & $(0.00003)$ & $(0.00025)$ & $(0.00021)$ & $(0.00117)$ \\
\hline \multirow{2}{*}{ EMU } & 0.03508 & 0.13376 & 0.83690 & -0.00159 & 0.04466 & 0.98078 & 0.13087 \\
& $(0.00055)$ & $(0.00108)$ & $(0.00139)$ & $(0.00009)$ & $(0.00047)$ & $(0.00045)$ & $(0.00106)$ \\
\hline \multirow{2}{*}{ OIL } & 0.03491 & 0.09306 & 0.86890 & -0.00294 & 0.04814 & 0.97390 & 0.11537 \\
& $(0.00048)$ & $(0.00075)$ & $(0.00111)$ & $(0.00010)$ & $(0.00042)$ & $(0.00049)$ & $(0.00106)$ \\
\hline \multirow{2}{*}{ GOLD } & 0.00194 & 0.02364 & 0.97441 & -0.00028 & 0.01676 & 0.99582 & 0.18268 \\
& $(0.00009)$ & $(0.00019)$ & $(0.00023)$ & $(0.00002)$ & $(0.00020)$ & $(0.00015)$ & $(0.00133)$ \\
\hline \multirow{2}{*}{ CRIX } & 0.13953 & 0.25253 & 0.63047 & 0.00926 & 0.17697 & 0.94151 & 0.40457 \\
& $(0.00109)$ & $(0.00138)$ & $(0.00180)$ & $(0.00047)$ & $(0.00088)$ & $(0.00062)$ & $(0.00147)$ \\
\hline \hline
\end{tabular}

The numbers in parentheses are asymptotic standard errors of the corresponding parameter estimates.

For the degrees-of-freedom parameter $\eta_{i}$ we estimate its inverse, i.e. $\bar{\eta}_{i}=\frac{1}{\eta_{i}} \in(0,1)$. 
Table 5: Volatility model diagnostics

\begin{tabular}{ccccccc}
\hline \hline & SPX & SBW & EMU & OIL & GOLD & CRIX \\
\hline score & 0.2206 & 0.6373 & 0.0893 & 0.5345 & 0.2228 & 0.8595 \\
$\varepsilon$ & 0.7973 & 0.4541 & 0.5860 & 0.2945 & 0.7376 & 0.0111 \\
$\varepsilon^{2}$ & 0.3428 & 0.0593 & 0.6779 & 0.5103 & 0.1769 & 0.0537 \\
\hline \hline
\end{tabular}

The table reports the p-values of Box-Pierce test applied to the score with respect to $\log$ volatility, the standardized residuals $(\varepsilon)$ and squared standardized residuals $\left(\varepsilon^{2}\right)$. The number of lags being tested is 30 .

Table 6: Descriptive statistics for residual terms

\begin{tabular}{ccccccc}
\hline \hline & SPX & SBW & EMU & OIL & GOLD & CRIX \\
\hline N.Obs & 835 & 835 & 835 & 835 & 835 & 835 \\
Minimum & -6.0863 & -4.2446 & -6.7650 & -4.1232 & -4.3351 & -6.0359 \\
Maximum & 3.3702 & 3.9371 & 3.7873 & 4.2435 & 4.4491 & 3.9927 \\
Mean & 0.0303 & -0.0061 & 0.0138 & 0.0031 & -0.0080 & 0.0199 \\
Stdev & 1.0277 & 1.0046 & 1.0109 & 1.0114 & 0.9889 & 0.8051 \\
Skewness & -1.1115 & -0.0884 & -0.4886 & -0.2190 & 0.0897 & -0.9780 \\
Kurtosis & 4.8534 & 0.8507 & 2.8784 & 1.1195 & 1.6104 & 8.4559 \\
\hline \hline
\end{tabular}

The kurtosis in the table is excess kurtosis. 
Table 7: Two-step parameter estimates for the correlation part of the model. The diagonal elements of $K$ and $\Phi$ are not restricted to be the same (Model 1).

\begin{tabular}{|c|c|c|c|c|c|}
\hline \multirow{7}{*}{$\omega$} & $\omega_{i * 5+1}$ & $\omega_{i * 5+2}$ & $\omega_{i * 5+3}$ & $\omega_{i * 5+4}$ & $\omega_{i * 5+5}$ \\
\hline & -0.03847 & 0.24809 & 0.02754 & -0.01034 & -0.00128 \\
\hline & $(0.00820)$ & $(0.14935)$ & $(0.17373)$ & $(0.01676)$ & $(0.00378)$ \\
\hline & 0.00616 & -0.00233 & 0.00012 & 0.00191 & 0.00108 \\
\hline & $(0.00206)$ & $(0.00779)$ & $(0.00866)$ & $(0.00682)$ & $(0.00581)$ \\
\hline & 0.00107 & 0.00615 & 0.04274 & 0.00144 & 0.00074 \\
\hline & $(0.01464)$ & $(0.02729)$ & $(0.03632)$ & $(0.00777)$ & $(0.00206)$ \\
\hline \multirow{7}{*}{$K$} & $\kappa_{i * 5+1}$ & $\kappa_{i * 5+2}$ & $\kappa_{i * 5+3}$ & $\kappa_{i * 5+4}$ & $\kappa_{i * 5+5}$ \\
\hline & 0.07631 & 0.05572 & 0.00042 & 0.01032 & 0.00032 \\
\hline & $(0.01135)$ & $(0.01365)$ & $(0.03423)$ & $(0.05389)$ & $(0.03428)$ \\
\hline & 0.05623 & 0.01455 & 0.01029 & 0.00697 & 0.01458 \\
\hline & $(0.01032)$ & $(0.04803)$ & $(0.01040)$ & $(0.03484)$ & $(0.00669)$ \\
\hline & 0.03884 & 0.00065 & 0.03662 & 0.01048 & 0.02749 \\
\hline & $(0.02710)$ & $(0.03378)$ & $(0.04887)$ & $(0.03798)$ & $(0.05471)$ \\
\hline \multirow{7}{*}{$\Phi$} & $\phi_{i * 5+1}$ & $\phi_{i * 5+2}$ & $\phi_{i * 5+3}$ & $\phi_{i * 5+4}$ & $\phi_{i * 5+5}$ \\
\hline & 0.79869 & 0.54849 & 0.88795 & 0.76492 & 0.87753 \\
\hline & $(0.05495)$ & $(0.26711)$ & $(0.71128)$ & $(0.53696)$ & $(0.62484)$ \\
\hline & 0.95936 & 0.94075 & 0.99975 & 0.91681 & 0.98965 \\
\hline & $(0.01769)$ & $(0.16792)$ & $(0.01582)$ & $(0.06843)$ & $(0.03547)$ \\
\hline & 0.87737 & 0.85322 & 0.52478 & 0.96373 & 0.95236 \\
\hline & $(0.04997)$ & $(0.68123)$ & $(0.25244)$ & $(0.07243)$ & $(0.02127)$ \\
\hline \multirow{2}{*}{$\bar{\eta}$} & 0.13435 & & & & \\
\hline & $(0.00822)$ & & & & \\
\hline
\end{tabular}

Standard errors are given in parentheses, and $i=0,1,2$. 
Table 8: Model descriptions

\begin{tabular}{ccc}
\hline \hline & DESCRIPTION & \# Pars \\
\hline Model 1 & full $\omega$, diagonal $K$, diagonal $\Phi$ & 46 \\
Model 2 & full $\omega$, scalar $K$, scalar $\Phi$ & 18 \\
Model 3 & scalar $\omega$, diagonal $K$, diagonal $\Phi$ & 32 \\
Model 4 & $\omega=0$, diagonal $K$, diagonal $\Phi$ & 31 \\
Model 5 & scalar $\omega$, scalar $K$, scalar $\Phi$ & 4 \\
Model 6 & $\omega=0$, scalar $K$, scalar $\Phi$ & 3 \\
\hline \hline
\end{tabular}

\# Pars is the number of parameters. 
Table 9: Diagnostics and model selection criteria for multivariate models

\begin{tabular}{ccccccc}
\hline \hline & Model 1 & Model 2 & Model 3 & Model 4 & Model 5 & Model 6 \\
\hline $\mathrm{k}$ & 46 & 18 & 32 & 31 & 4 & 3 \\
$\mathrm{~T}$ & 835 & 835 & 835 & 835 & 835 & 835 \\
score test & 0.5095 & 0.3422 & 0.4867 & 0.4672 & 0.4438 & 0.4429 \\
$-\ln \mathcal{L}_{i}(\hat{\theta})$ & 6507.10 & 6529.30 & 6520.04 & 6526.95 & 6562.65 & 6563.24 \\
$\mathrm{LR}$ & & 44.39 & 25.88 & 39.71 & 111.10 & 112.29 \\
d.o.f. & & 28 & 14 & 15 & 42 & 43 \\
p-value & & 0.0254 & 0.0268 & 0.0005 & 0.0000 & 0.0000 \\
BIC & 13323.66 & 13179.68 & 13255.36 & 13262.45 & 13152.20 & 13146.67 \\
AIC & 13106.20 & 13094.59 & 13104.08 & 13115.90 & 13133.29 & 13132.49 \\
\hline \hline
\end{tabular}

$\mathrm{k}$ is the number of parameters in the model.

$\mathrm{T}$ is the number of data points in the sample.

score test is the p-value of a multivariate portmanteau test applied to the correlation scores with number of lags 30 and degree of freedom $N_{\text {lag }} * N^{* 2}$, with $N_{\text {lag }}=30$, and $N^{*}=15$.

$L R=-2 \ln \frac{\mathcal{L}_{i}(\hat{\theta})}{\mathcal{L}_{1}(\hat{\theta})}$, with $\mathcal{L}_{i}$ the likelihood for model i.

Degree of freedom is difference in the number of parameters for the model with respect to Model 1.

$B I C=-2 \ln \mathcal{L}(\hat{\theta})+k \ln T$

$A I C=-2 \ln \mathcal{L}(\hat{\theta})+2 k$ 

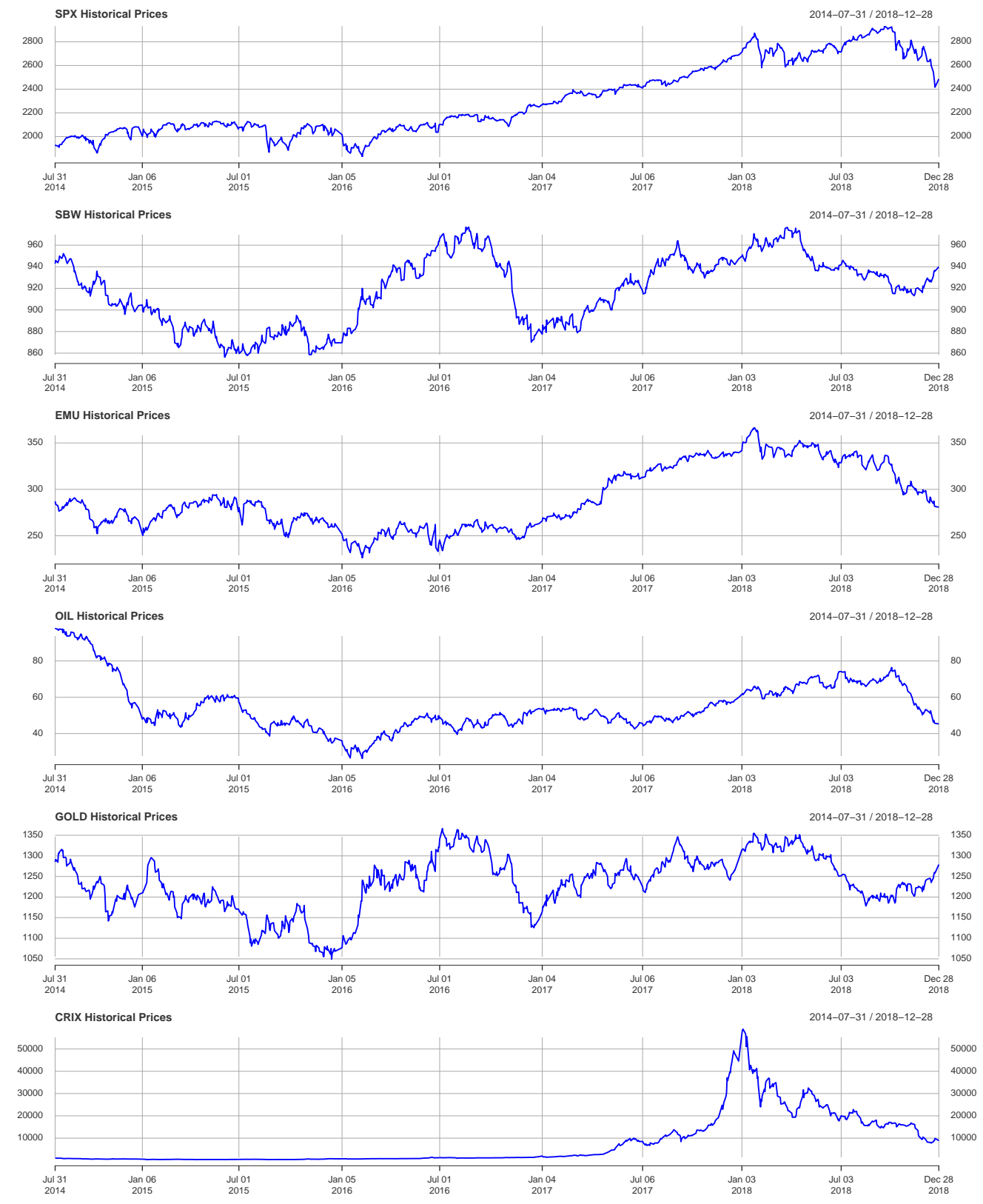

Figure 2: Time series of daily asset prices, August 1, 2014 to December 28, 2018, for (from top to bottom) SPX, SBW, EMU, OIL, GOLD, and CRIX. 

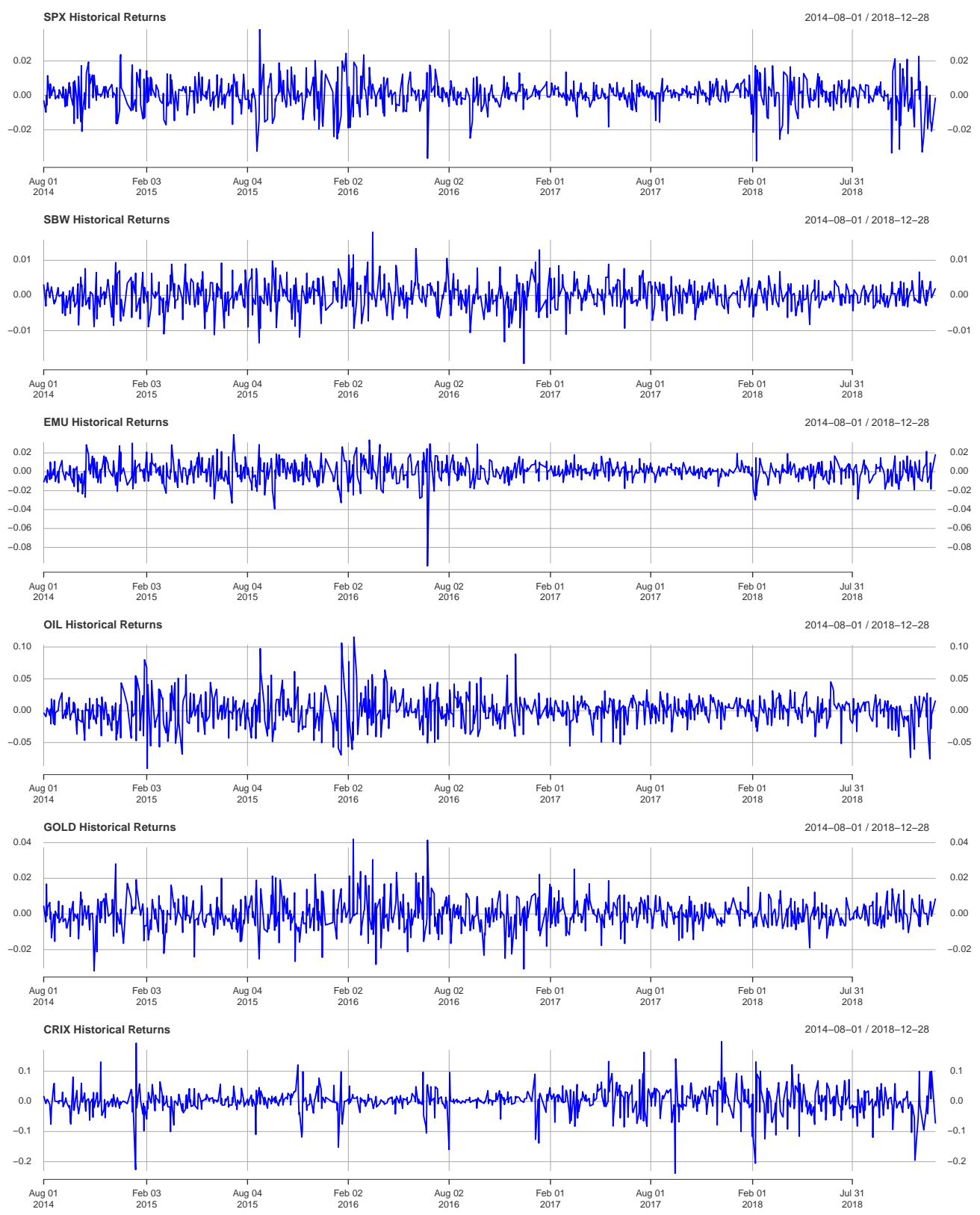

Figure 3: Time series of daily returns, August 1, 2014 to December 28, 2018, for (from top to bottom) SPX, SBW, EMU, OIL, GOLD, and CRIX. 

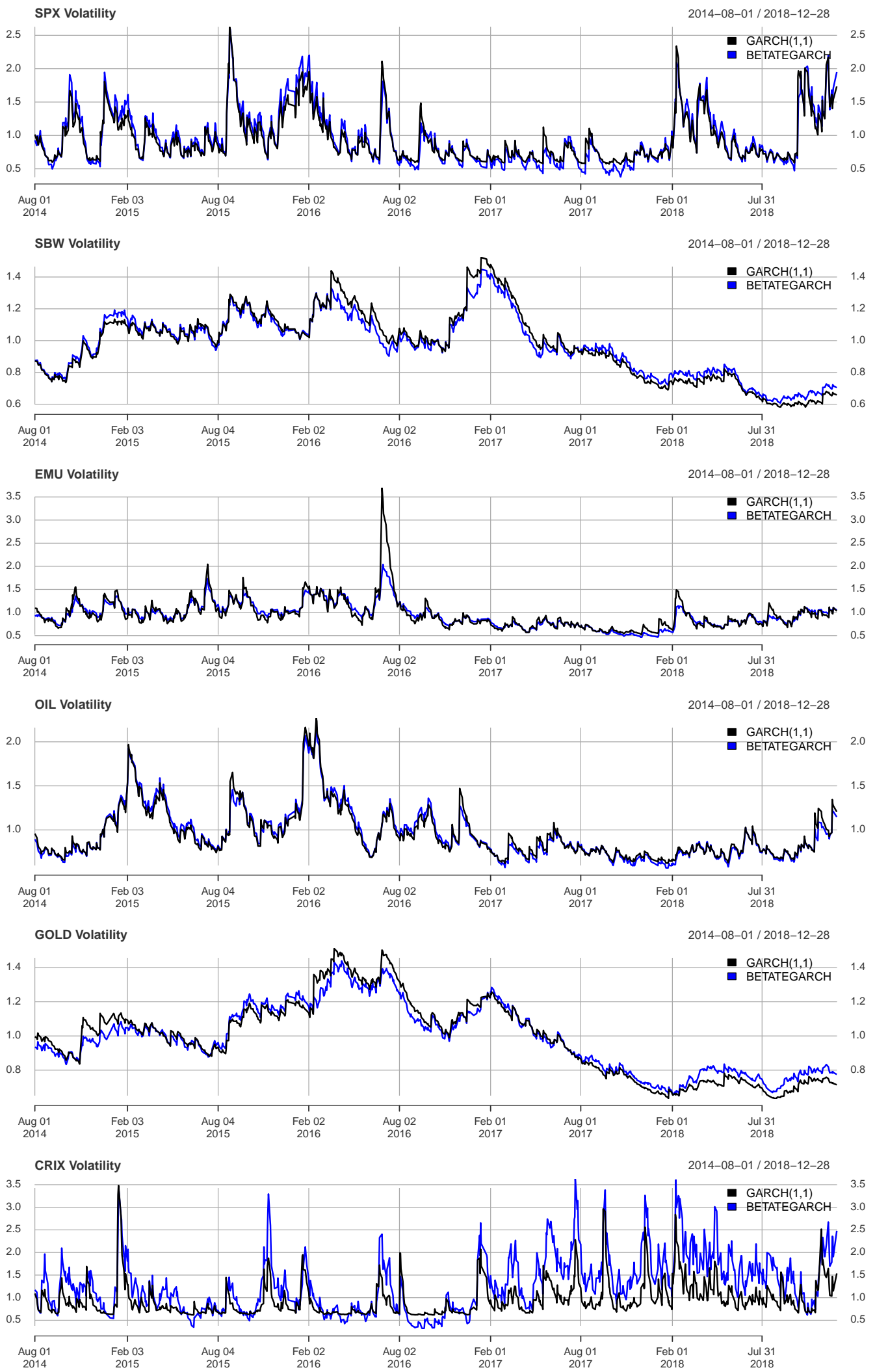

Figure 4: Estimated volatility series using GARCH(1,1) and Beta-t-EGARCH. 

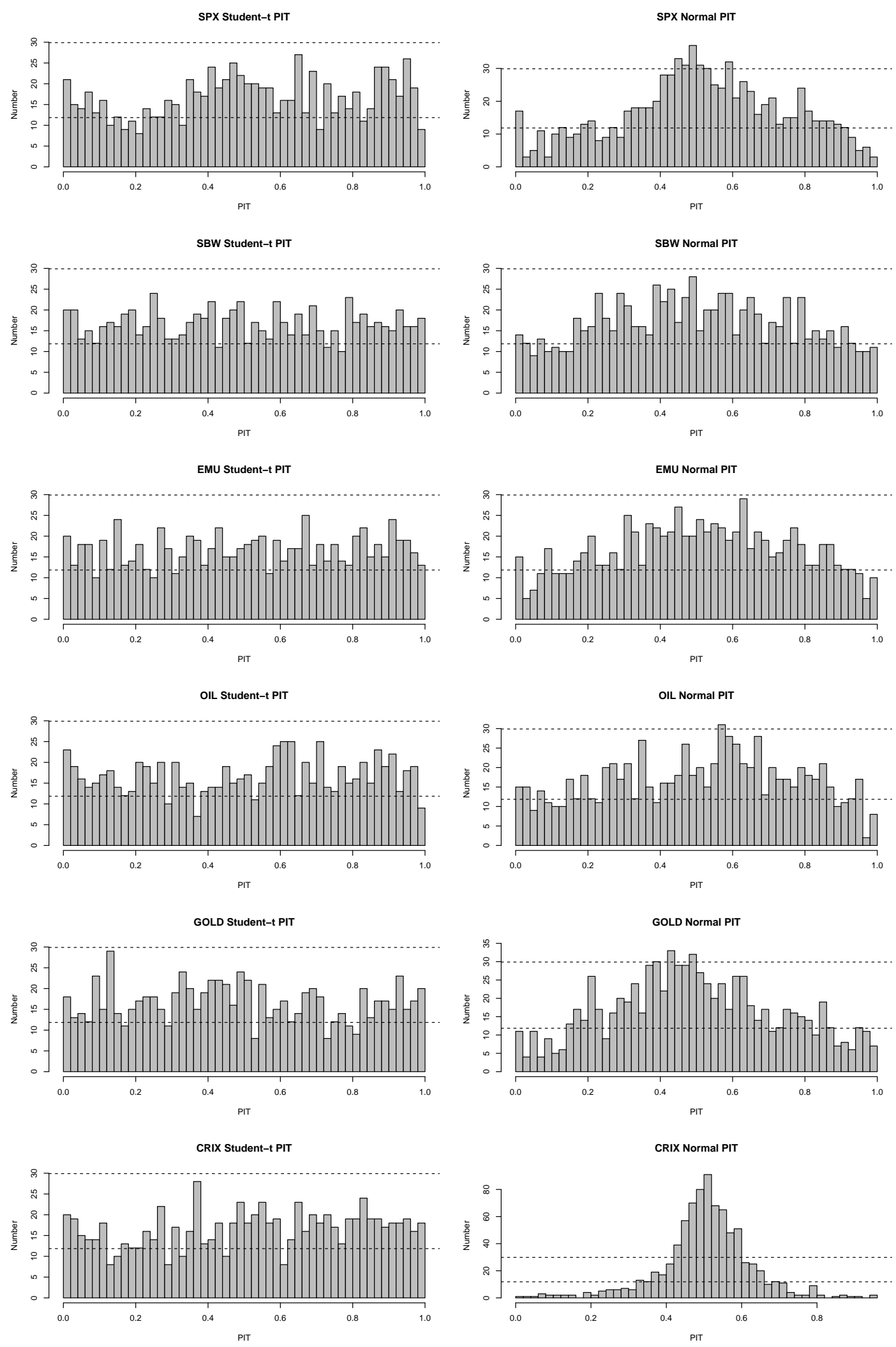

Figure 5: Histograms of probability integral transforms (PITs) of standardized residuals of the Beta-t-EGARCH model. For the figures on the right, the Gaussian cdf is used for the transformation, and on the left the student-t distribution with the estimated d.o.f.. 

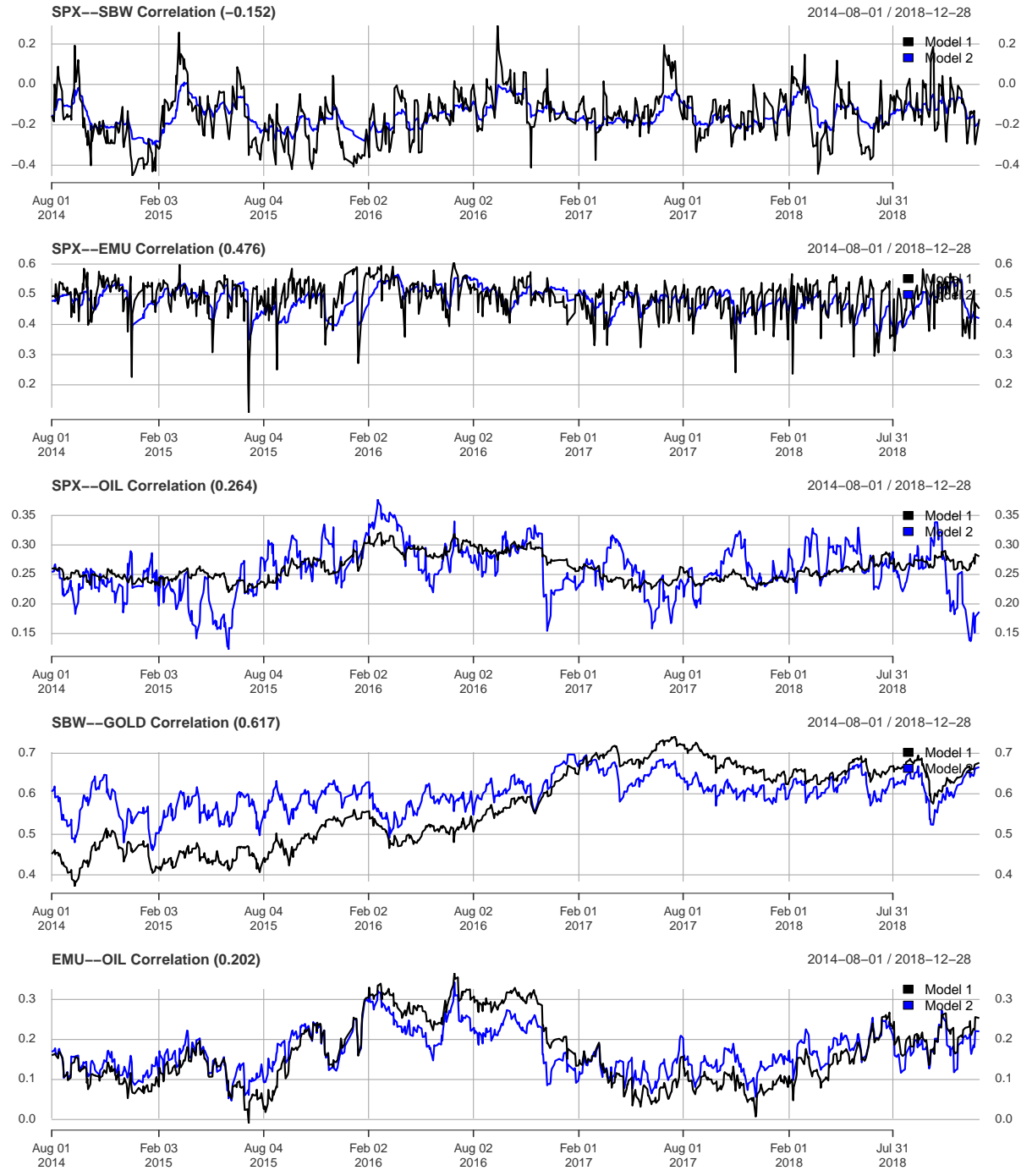

Figure 6: Estimated conditional correlation series for five selected pairs (from top to bottom): SPX-SBW, SPX-EMU, SPX-OIL, SBW-GOLD, EMU-OIL, using two models: Model 1 has no restriction for the diagonal elements of $K$ and $\Phi$, while Model 2 restricts them to be equal. 


\section{References}

Archakova, I. And P. R. Hansen (2018): "A new parametrization of correlation matrices". Working Paper.

Bauwens, L, C. Hafner And S. Laurent (2012): Handbook of volatility models and their applications. Wiley.

Baumens, L. And S. Laurent (2005): "A New Class of Multivariate Skew Densities, With Application to Generalized Autoregressive Conditional Heteroscedasticity Models". Journal of Business \& Economic Statistics 23.3, pp. 346-354.

Bauwens, L., S. Laurent and J. V. Rombouts (2006): "Multivariate GARCH models: a survey". Journal of Applied Econometrics 21.1, pp. 79-109.

Brown, B. M. (1971): "Martingale Central Limit Theorems". Ann. Math. Statist. 42.1, pp. 59-66.

CAporin, M. And M. MCAleER (2012): "Do we really need both BEKK and DCC? A tale of two multivariate GARCH models". Journal of Economic Surveys 26.4, pp. 736751.

Creal, D., S. J. Koopman And A. Lucas (2011): "A dynamic multivariate heavytailed model for time-varying volatilities and correlations". Journal of Business \& Economic Statistics 29.4, pp. 552-563.

Creal, D., S. J. Koopman And A. Lucas (2013): "Generalized autoregressive score models with applications". Journal of Applied Econometrics 28.5, pp. 777-795.

Demarta, S. And A. J. MCNeil (2005): "The t Copula and Related Copulas". International Statistical Review 73.1, pp. 111-129.

Engle, R. F. (2002): "Dynamic Conditional Correlation: A Simple Class of Multivariate Generalized Autoregressive Conditional Heteroskedasticity Models". Journal of Business \& Economic Statistics 20.3, pp. 339-350.

Fiorentini, G., E. Sentana and G. Calzolari (2003): "Maximum likelihood estimation and inference in multivariate conditionally heteroscedastic dynamic regression 
models with Student t innovations". Journal of Business 83 Economic Statistics 21.4, pp. $532-546$.

FrANCQ, C. AND J.-M. ZAKOIAN (2019): GARCH models: structure, statistical inference and financial applications. John Wiley \& Sons.

Gorgi, P, P. Hansen, P Janus and S. Koopman (2018): "Realized wishart-garch: a score-driven multi-asset volatility model". Journal of Financial Econometrics 17.1, pp. $1-32$.

Hafner, C. M., O. B. Linton and H. Tang (2019): "Estimation of a Multiplicative Correlation Structure in the Large Dimensional Case". Journal of Econometrics, forthcoming.

Harvey, A. And R.-J. Lange (2017): "Volatility Modeling with a Generalized t Distribution". Journal of Time Series Analysis 38.2, pp. 175-190.

Harvey, A. and G. SuCARrat (2014): "EGARCH models with fat tails, skewness and leverage". Computational Statistics \& Data Analysis 76, pp. 320 -338.

Harvey, A. C. (2013): Dynamic models for volatility and heavy tails: with applications to financial and economic time series. Vol. 52. Cambridge University Press.

Jensen, S. T. AND A. RAhbek (2004): "ASYMPTOTIC INFERENCE FOR NONSTATIONARY GARCH". Econometric Theory 20.6, 1203-1226.

Joe, H. (1997): Multivariate Models and Dependence Concepts. Chapman \& Hall.

JoE, H. (2005): "Asymptotic efficiency of the two-stage estimation method for copulabased models". Journal of Multivariate Analysis 94, pp. 401-419.

KaWAKatsu, H. (2006): "Matrix exponential GARCH". Journal of Econometrics 134.1, pp. $95-128$.

Nelson, D. B. (1991): "Conditional Heteroskedasticity in Asset Returns: A New Approach". Econometrica 59.2, pp. 347-370.

Opschoor, A., P. Janus, A. Lucas and D. V. Dijk (2018): "New HEAVY Models for Fat-Tailed Realized Covariances and Returns". Journal of Business $\& 3$ Economic Statistics 36.4, pp. 643-657. 
Straumann, D. and T. Mikosch (2006): "Quasi-maximum-likelihood estimation in conditionally heteroscedastic time series: A stochastic recurrence equations approach". Ann. Statist. 34.5, pp. 2449-2495.

Trimborn, S. ANd W. K. HärdLe (2018): "CRIX an Index for cryptocurrencies". Journal of Empirical Finance 49, pp. 107 -122.

VAssallo, D., G. BuCCheri and F. Corsi (2018): "A DCC-type approach for Realized Covariance modelling with score-driven dynamics". Available at SSRN 3305628. 